
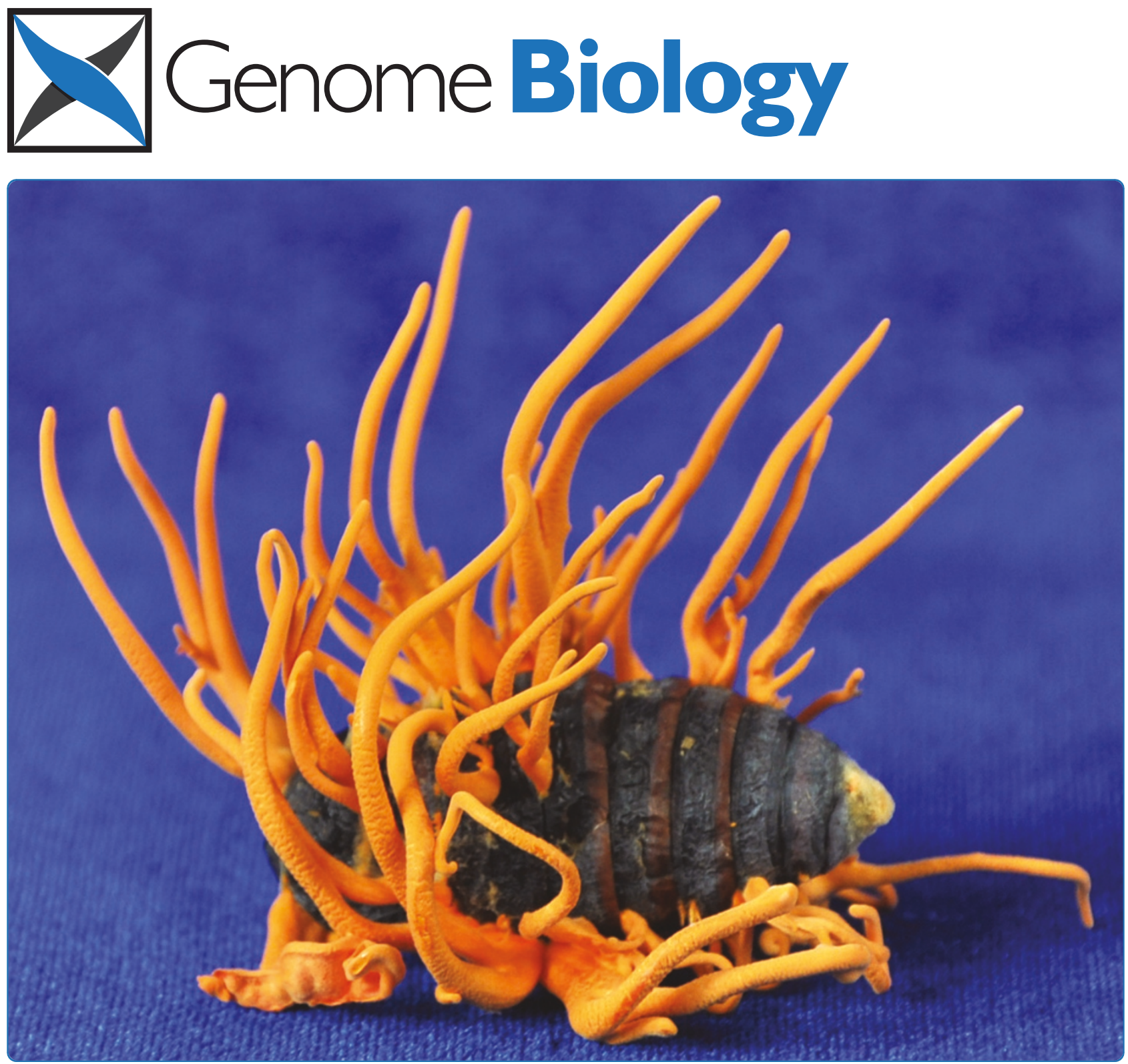

Genome sequence of the insect pathogenic fungus Cordyceps militaris, a valued traditional chinese medicine

Zheng et al.

() Biomed Central

Zheng et al. Genome Biology 2011, 12:R116

http://genomebiology.com/2011/12/11/R116 (23 November 2011) 


\title{
Genome sequence of the insect pathogenic fungus Cordyceps militaris, a valued traditional chinese medicine
}

\author{
Peng Zheng ${ }^{1 \dagger}$, Yongliang Xia ${ }^{1 \dagger}$, Guohua Xiao ${ }^{1 \dagger}$, Chenghui Xiong ${ }^{1}$, Xiao Hu${ }^{1}$, Siwei Zhang ${ }^{1}$, Huajun Zheng ${ }^{2}$, \\ Yin Huang ${ }^{2}$, Yan Zhou', Shengyue Wang ${ }^{2}$, Guo-Ping Zhao ${ }^{1,2}$, Xingzhong Liu ${ }^{3}$, Raymond J St Leger ${ }^{4}$ and \\ Chengshu Wang ${ }^{1 *}$
}

\begin{abstract}
Background: Species in the ascomycete fungal genus Cordyceps have been proposed to be the teleomorphs of Metarhizium species. The latter have been widely used as insect biocontrol agents. Cordyceps species are highly prized for use in traditional Chinese medicines, but the genes responsible for biosynthesis of bioactive components, insect pathogenicity and the control of sexuality and fruiting have not been determined.

Results: Here, we report the genome sequence of the type species Cordyceps militaris. Phylogenomic analysis suggests that different species in the Cordyceps/Metarhizium genera have evolved into insect pathogens independently of each other, and that their similar large secretomes and gene family expansions are due to convergent evolution. However, relative to other fungi, including Metarhizium spp., many protein families are reduced in C. militaris, which suggests a more restricted ecology. Consistent with its long track record of safe usage as a medicine, the Cordyceps genome does not contain genes for known human mycotoxins. We establish that C. militaris is sexually heterothallic but, very unusually, fruiting can occur without an opposite mating-type partner. Transcriptional profiling indicates that fruiting involves induction of the Zn2Cys6-type transcription factors and MAPK pathway; unlike other fungi, however, the PKA pathway is not activated.
\end{abstract}

Conclusions: The data offer a better understanding of Cordyceps biology and will facilitate the exploitation of medicinal compounds produced by the fungus.

\section{Background}

The Ascomycete genus Cordyceps includes over 500 species that are pathogens of arthropods. Cordyceps militaris (CCM) is the type species and occurs throughout much of the Northern Hemisphere as a pathogen of lepidopteran insect pupae [1]. C. militaris is readily characterized by the sexual fruiting bodies forming on mycosed pupae, the structures giving the fungus its common name of 'pupa grass' in China. Anamorphic Cordyceps species, such as Beauveria spp., Metarhizium spp. and Paecilomyces spp., have been developed as

\footnotetext{
* Correspondence: cswang@sibs.ac.cn

+ Contributed equally

${ }^{1}$ Key Laboratory of Insect Developmental and Evolutionary Biology, Institute of Plant Physiology and Ecology, Shanghai Institutes for Biological Sciences, Chinese Academy of Sciences, 300 Fenglin Road, Shanghai 200032, China Full list of author information is available at the end of the article
}

insect biocontrol agents [2,3]. Although C. militaris and Cordyceps sinensis (syn. Ophiocordyceps sinensis) are best known as traditional Chinese medicines, they are also increasingly being studied and used in the West $[4,5]$. An array of pharmacologically active components has been identified, including cordycepin, cordycepic acids, polysaccharides and macrolides [6]. Cordycepin (3'-deoxyadenosine) has so far only been reported in $C$. militaris and is a broad spectrum antimicrobial [5] and polyadenylation inhibitor that is currently undergoing clinical trials against cancers [7]. The biosynthetic pathway of cordycepin production has not been determined.

In spite of their market values - for example, > $\$ 10,000$ per kilo for the fruiting bodies of the un-cultivatable C. sinensis [8] - very little is known about sex and developmental processes in Cordyceps species, and remedying this deficiency should help in production/

\section{Biomed Central}


cultivation of these enigmatic fungi. C. militaris is notable as it readily performs sexual reproduction on artificial media and is thus a good target for studying the molecular underpinnings of sex and development in Cordyceps spp. (Figure 1). C. militaris also has the potential to be a versatile new model for studying the evolution of sex and reproductive structures. While current fungal models have provided numerous insights into the evolution of sex [9], there is still much to be understood about the mechanisms, evolution and ecological impact of sexuality in fungi. This is, in part, because fungal mating and sexual cycles are often complicated; for example, aspergilli have both self-fertile (homothallism) and self-sterile (heterothallism) mating systems [10].

There is also much to be learnt about the nature and evolution of interactions of Cordyceps spp. with their hosts and with the wider environment. As entomopathogenicity appears to have evolved independently in Cordyceps and two Metarhizium species [11], comparative genomics will provide independent assessments of what is required to be entomopathogenic, identify the degree to which evolution between these fungi has been convergent, and identify the genomic basis of their differing physiologies and host-specificity. Last but not least, genomic sequencing of $C$. militaris will enable a systematic exploration of the biology and pharmaceuticals underlying the widespread medical impact of Cordyceps spp., and identify potential safety hazards, including genes for known human mycotoxins.

\section{Results}

\section{Genome sequencing and general features}

The C. militaris genome was shotgun sequenced to 147 $\times$ coverage and assembled into 33 scaffolds with an N50 of $4.6 \mathrm{Mb}$ and a total genome size of $32.2 \mathrm{Mb}$. The genome is smaller than either the broad host range Metarhizium anisopliae (MAA) or the locust-specific pathogen Metarhizium acridum (MAC) that we sequenced previously (Table 1 ). The characteristic telomeric repeats (TTAGGG/CCCTAA) $n$ were found at either 5' or 3' terminal of 13 scaffolds, including the terminal anchoring of two scaffolds, that is, the complete chromosomes. From mapping > 5,000 expressed sequence tags [12], the $C$. militaris genome was estimated to be $>99 \%$ complete. The genome was predicted to encode 9,684 protein genes, which is slightly fewer than $M$. anisopliae and M. acridum (Table 1). Consequently, many protein functional categories are smaller in Cordyceps than in Metarhizium spp. (Figure 2a). However, like M. anisopliae (17.6\%) and M. acridum (15.1\%), C. militaris has a higher proportion of its genes encoding putatively secreted proteins $(15.9 \%)$ than other sequenced ascomycetes (5 to $10 \%)[10,13,14]$.

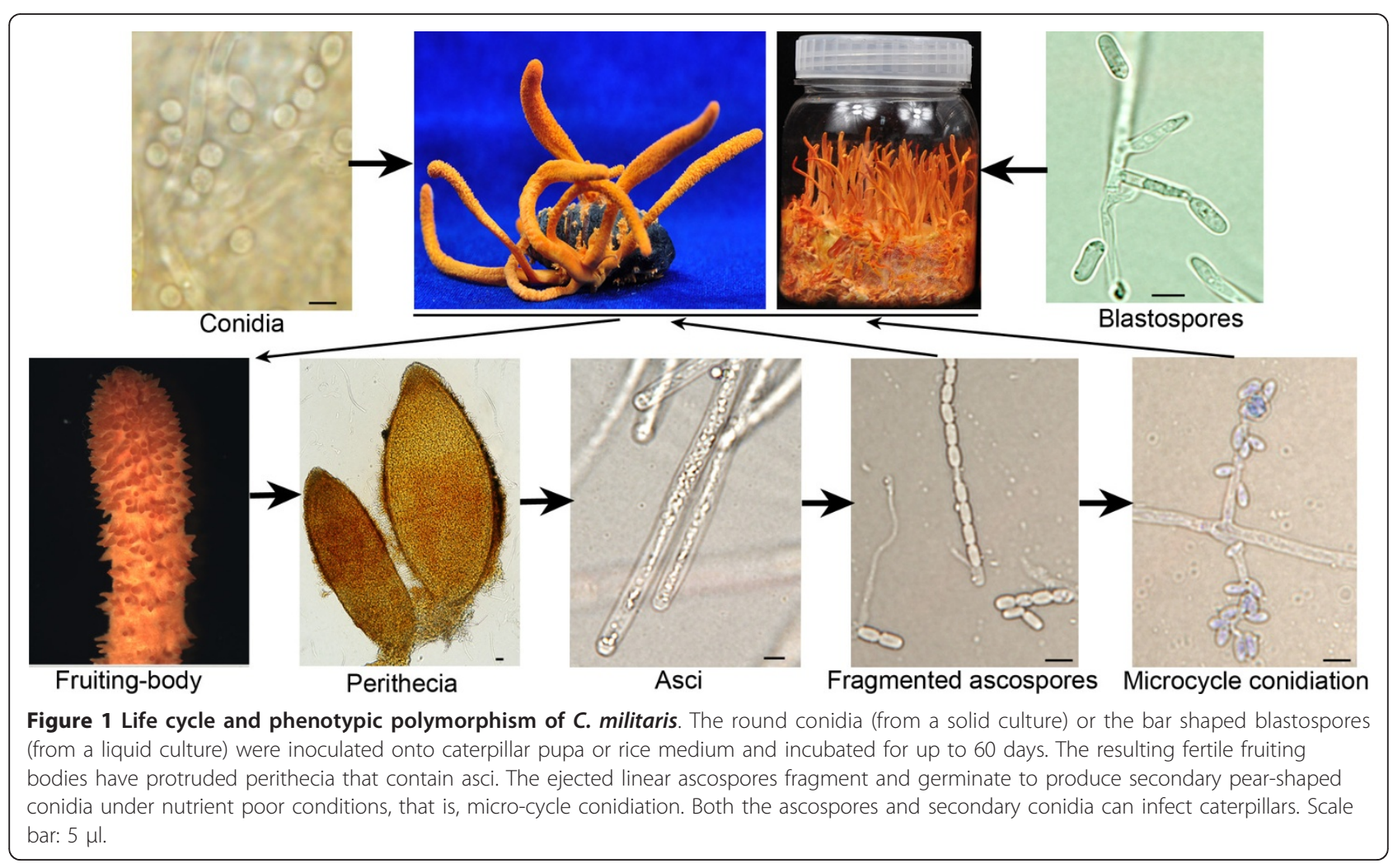


Table 1 Comparison of genome features among three insect pathogens

\begin{tabular}{lccc}
\hline Features & C. militaris & M. anisopliae & M. acridum \\
\hline Size (Mb) & 32.2 & 39.0 & 38.1 \\
Coverage (fold) & $147 \times$ & $100 \times$ & $107 \times$ \\
$\begin{array}{l}\text { Percentage G+C } \\
\text { content }\end{array}$ & 51.4 & 51.5 & 50.0 \\
$\begin{array}{l}\text { Percentage repeat rate } \\
\text { Protein-coding genes }\end{array}$ & 3.04 & 0.98 & 1.52 \\
Gene density (genes & 9,684 & 10,582 & 9,849 \\
per Mb) & 257 & 271 & 259 \\
Exons per gene & 3.0 & 2.8 & 2.7 \\
$\begin{array}{l}\text { Percentage secreted } \\
\text { proteins }\end{array}$ & 16.2 & 17.6 & 15.1 \\
tRNA & 136 & 141 & 122 \\
Pseudogenes & 102 & 363 & 440 \\
NCBI accession & AEV 00000000 & ADNJ00000000 & ADNI00000000 \\
\hline
\end{tabular}

$\mathrm{Mb}$, mega base pairs.

An InterproScan analysis identified 2,736 conserved protein families in C. militaris (containing 6,725 proteins), fewer than those in M. anisopliae (7,556 proteins in 2,796 families) or M. acridum (6,948 proteins in 2,746 families) [11]. In particular, the number of transposases is much fewer in C. militaris (4) than in Metarhizium spp. (148 in M. anisopliae and 20 in $M$. acridum) or other sequenced ascomycetes (15 to 426) (Table S1 in Additional file 1). The C. militaris genome lacks retrotransposase (Table S2 in Additional file 1), and has more than three-fold fewer pseudogenes than Metarhizium spp. (Table S3 in Additional file 1). About $16 \%$ of the predicted C. militaris genes $(1,547)$ are putatively involved in pathogen-host interactions; this proportion is slightly lower than for Metarhizium spp. (17.3\% in MAA and $16.5 \%$ in MAC) but is higher than four plant pathogens (10.8 to $15.5 \% ; P=0.0476$; false discovery rate $(\mathrm{FDR})=0.0152)$ (Table S4 in Additional file 1).

More than $50 \%$ of M. anisopliae and M. acridum proteins have $>90 \%$ identity [11]. Although the rarely observed sexual stages of Metarhizium spp. have been identified as a Cordyceps species [1], the analysis revealed that $<2 \%$ of $C$. militaris genes were highly conserved in comparison with those from Metarhizium spp., that is, had Blast score ratio (BSR) values close to 1 (Figure 3a). A similar pattern was observed when comparing C. militaris, M. anisopliae and the plant pathogen Fusarium graminearum (Figure 3b). Comparative genomic analysis of the three insect pathogens found that the percentage of species-specific genes is much higher in C. militaris (13.7\%) compared to $M$. anisopliae (4.8\%) and M. acridum (3.5\%) (Figure 2b). Based on the identities between orthologous proteins, $C$. militaris displays an average of approximately 63\% amino acid identity with either M. anisopliae or M. acri$d u m$, slightly higher than with the plant pathogens $F$. graminearum (61.6\%) and Magnaporthe oryzae (56.0\%) (Table 2). Thus, the three insect pathogenic fungi are more highly diverged than $F$. graminearum, Fusarium oxysporum and Fusarium verticillioides, which share an average of $85 \%$ nucleotide sequence identity [14], and Aspergillus nidulans, Aspergillus fumigatus and Aspergillus oryzae, which share an average of $68 \%$ amino acid sequence identity [13], and Trichoderma reesei, Trichoderma virens and Trichoderma atroviride, which share an average of $>70 \%$ amino acid sequence identity [15].

The regions containing at least three contiguous open reading frames that are not present in the reference genome are designated as genomic islands (GIs) [16]. Whole genome reciprocal analysis of three insect pathogens demonstrated that, in comparison to Metarhizium spp., C. militaris has 52 GIs (2\% coverage of its genome, harboring $21 \%$ of its species-specific genes), which is many more than $M$. anisopliae (8 GIs, $0.3 \%$ ) or M. acridum (5 GIs, $0.2 \%$ ) when referenced to C. militaris. As in aspergilli [17], many C. militaris species-specific gene-encoding proteins do not have conserved domains and the genes are clustered together to form GIs (Table 2). A phylogenomic analysis established that the Cordyceps lineage is more closely related to the wheat pathogen F. graminearum (divergence time of 200 to 260 million years ago (MYA)) than it is to Metarhizium spp. (26 to 34 MYA) (Figure 3c). Thus, the lineage leading to C. militaris appears to have diverged from plant pathogens around the Triassic-Jurassic boundary (200 MYA), while $M$. anisopliae and $M$. acridum diverged after the Cretaceous Extinction Event (65 MYA) [18]. Analysis of paralogous genes found only one pair of C. militaris genes with $>90 \%$ nucleotide sequence similarities (Figure 3d), which is similar to Neurospora crassa (one pair) [13] and F. graminearum (two pairs) [19]. Analysis of 24 paired C. militaris genes showing $>70 \%$ nucleotide identities found a strong overall C:G to T:A mutation bias (Figure S1 in Additional file 2), consistent with repeat-induced point mutations, the DNA methylationlinked processes that cause mutations of repeated fungal sequences $[15,20]$.

\section{Protein family analysis}

We identified gene family expansions for proteases, chitinases, lipases and protein kinases in C. militaris when compared with phytopathogenic fungi, whereas gene family contractions occurred for glycoside hydrolases (GHs; $P=0.0144$; FDR $=0.02)$, cutinases $(P=0.0065$; FDR $=0.0226)$ and pectin lyases $(P=0.0245$; FDR $=$ 0.0284) (Table S1 in Additional file 1). The largest family expansions were for proteases. The $C$. militaris genome contains 61 families of proteases but most of 


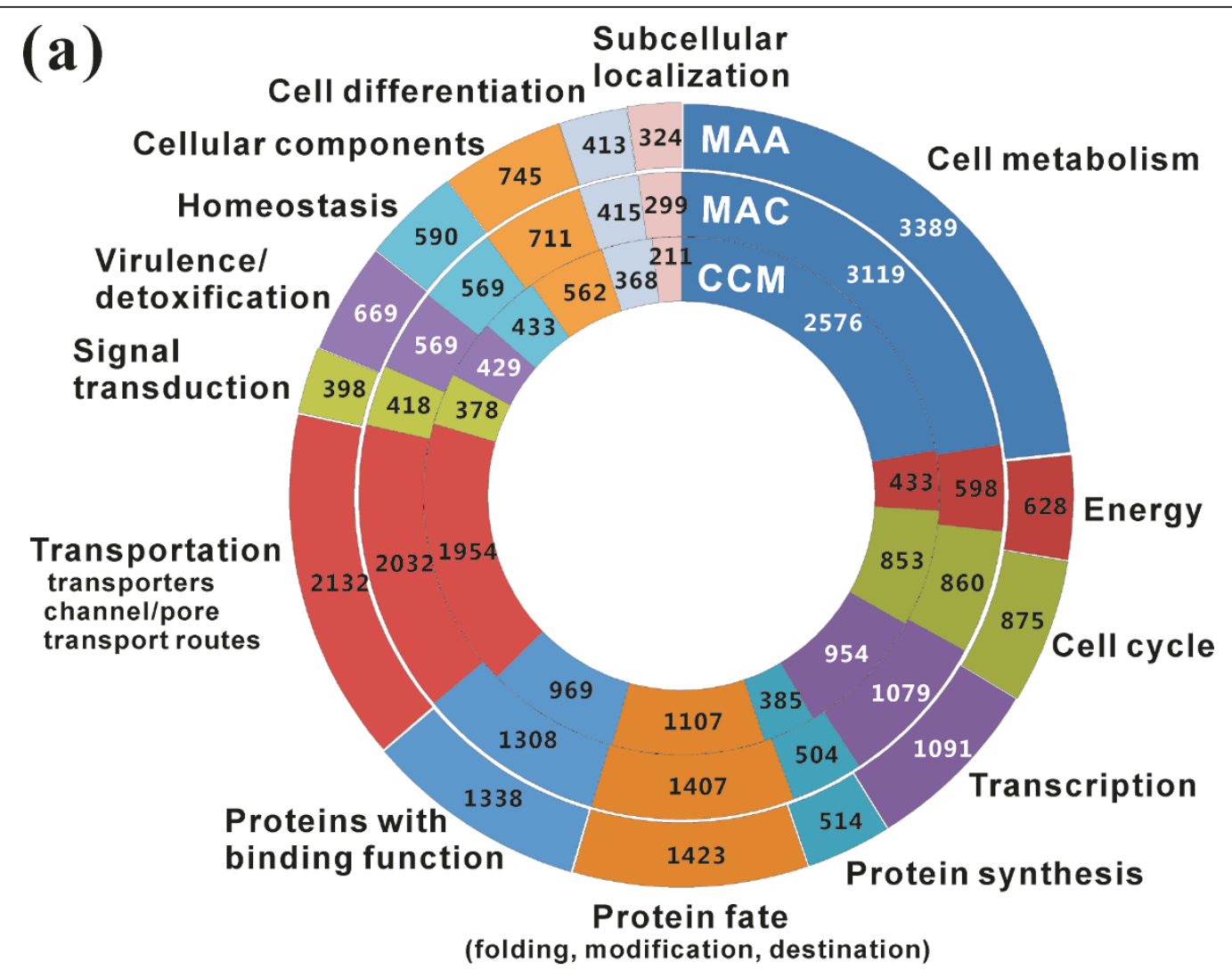

(b)

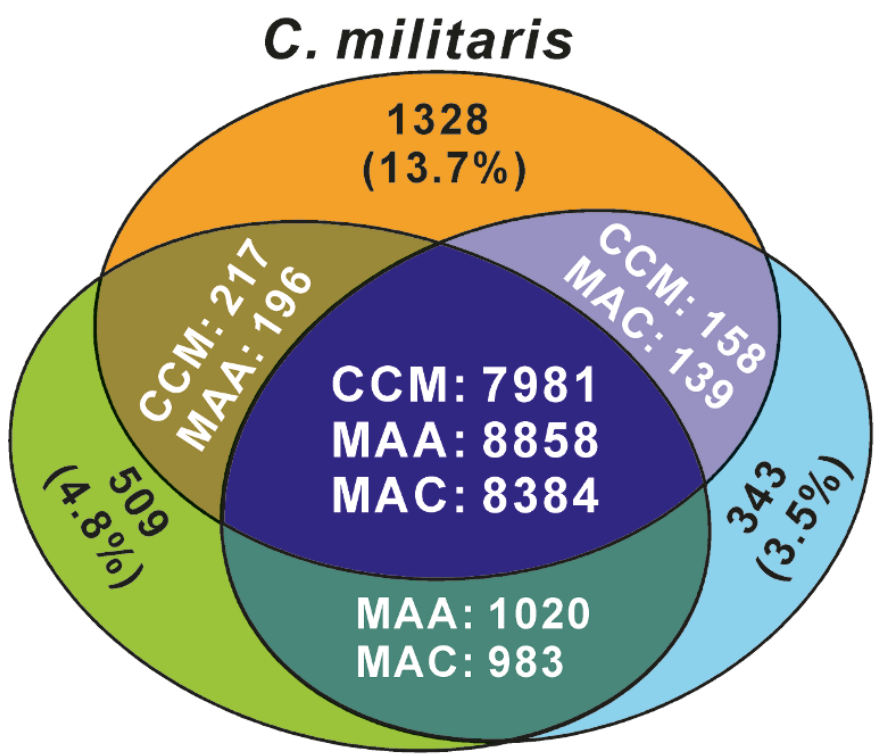

\section{M. anisopliae}

M. acridum

Figure 2 Comparative genomics analysis of three insect pathogens. (a) Functional classification and comparison of C. militaris (CCM), M. anisopliae (MAA) and M. acridum (MAC) proteins, showing that C. militaris has fewer genes in each category. Each circle represents the relative fraction of genes represented in each of the categories for each genome. (b) Reciprocal blast analysis of the predicted proteins among three insect pathogens. The cut-off $E$ value is at $\leq 1 e-5$. 


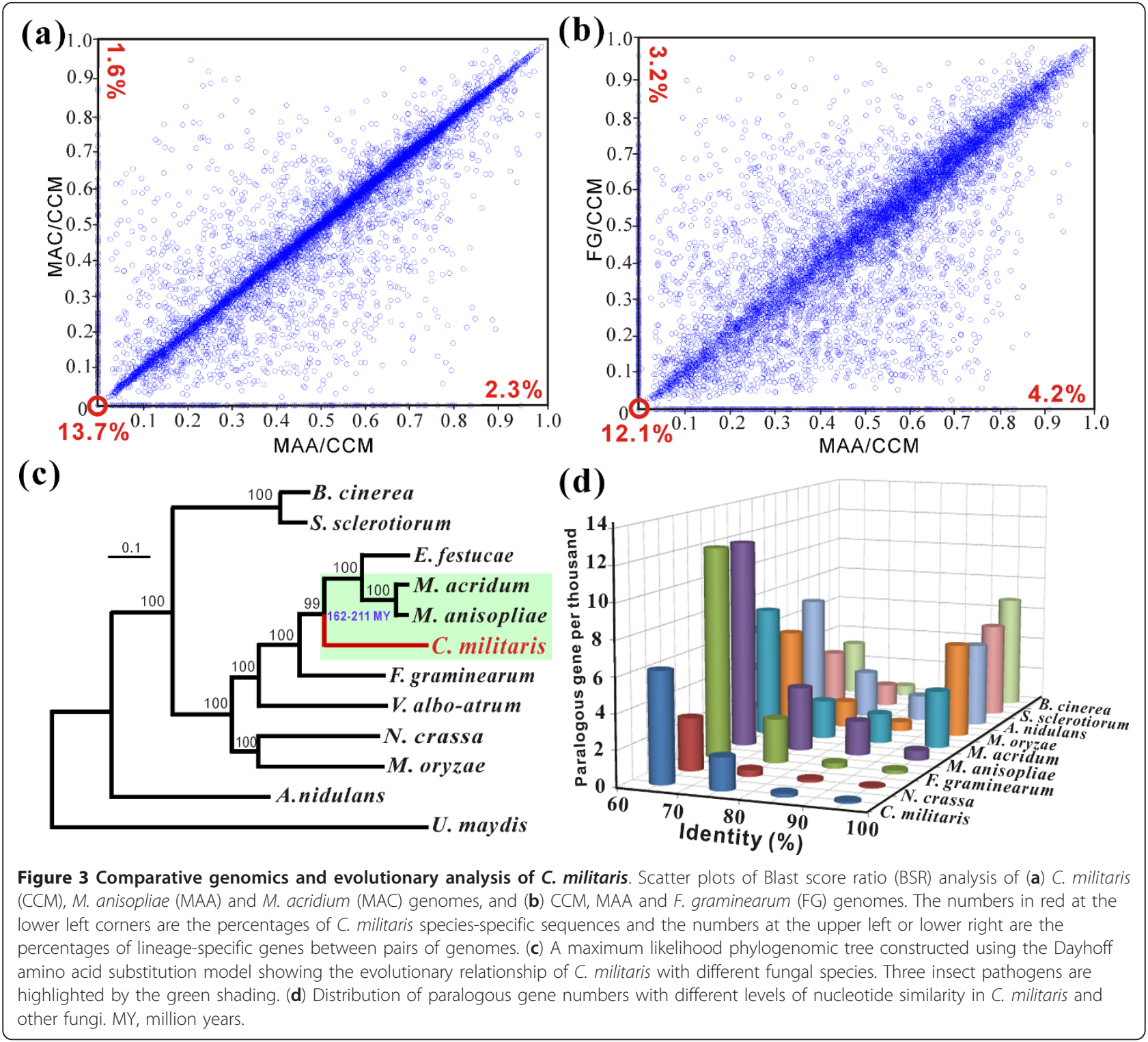

them were included in families of serine proteases (180/ $381)$ and metallopeptidases (108/381) (Table S5 in Additional file 1). Gene expansions within the subtilisin $(P=$ 0.0109; FDR $=0.0189)$ and trypsin $(P=0.0077$; FDR $=$ 0.0178 ) families are consistent with their being virulence factors in insect pathogens [11]. However, different families of proteases are expanded in Metarhizium spp. and $C$. militaris, consistent with each lineage 'reinventing the wheel' during the evolution of entomopathogenicity. Thus, relative to Metarhizium spp., the S01 trypsin and S08 subtilisin subfamilies are smaller and the S53 subfamily is larger (Table S6 in Additional file 1). The C. militaris genome has 12 trypsin genes compared to 4 or less in plant pathogens. It lacks four subfamilies of trypsins present in M. anisopliae. Interestingly, the bacterial-like chymotrypsin identified in $M$. anisopliae [21] is absent in $M$. acridum [11] but present as two copies in C. militaris. The A01 aspartyl proteases are virulence factors of both mammalian and plant pathogens because of their ability to cleave an array of host proteins [22]. Compared to phytopathogenic fungi (average 17), their number is significantly $(P=0.0059$; FDR $=$ 0.0057 ) expanded in the three insect pathogens (average 24) (Table S4 in Additional file 1).

Compared to many plant pathogens, Metarhizium spp. and C. militaris have fewer cutinases for degrading plant cell walls (Table S1 in Additional file 1). They also have fewer (average 137, $P<0.05$ ) GHs than plant pathogens (average 199), including the lack of $20 \mathrm{GH}$ families used by most plant pathogens and saprobes to target plant 
Table 2 Genome-wide analysis of $C$. militaris gene sets

\begin{tabular}{|c|c|c|c|c|c|}
\hline Characteristics & C. militaris & CMM core $^{a}$ & CMA restricted ${ }^{\mathbf{b}}$ & CMC restricted ${ }^{c}$ & CCM specific \\
\hline Number of genes & 9,684 & 7,981 & 217 & 158 & 1,328 \\
\hline Mean gene length (bp) & 1,742 & 1,885 & 1,445 & 1,440 & 967 \\
\hline Mean number of introns per gene & 1.99 & 2.05 & 1.77 & 1.69 & 1.71 \\
\hline Percentage genes without introns & 21.3 & 20.1 & 22.6 & 28.5 & 27.5 \\
\hline Percentage GC content (excluding introns) & 58.6 & 58.6 & 70.7 & 58.7 & 58.3 \\
\hline Number of InterproScan protein families & 2,644 & 2,552 & 69 & 52 & 112 \\
\hline Number of secreted proteins & 1,572 & 1,250 & 45 & 13 & 264 \\
\hline Number of PHI genes ${ }^{d}$ & 1,547 & 1,539 & 4 & 2 & 2 \\
\hline Number of TSA proteases & 68 & 65 & 3 & 0 & 0 \\
\hline Number of MFS genes & 245 & 242 & 0 & 2 & 1 \\
\hline Number of cytochrome P450s & 57 & 56 & 0 & 1 & 0 \\
\hline Number of Pth11-like GPCRs & 18 & 18 & 0 & 0 & 0 \\
\hline Number of protein kinases & 167 & 167 & 0 & 0 & 0 \\
\hline Number of transcription factors & 123 & 120 & 2 & 1 & 0 \\
\hline Number of glycoside hydrolases & 105 & 103 & 2 & 0 & 0 \\
\hline Number of SM backbone genes & 28 & 28 & 0 & 0 & 0 \\
\hline Number of horizontally transferred genes & 49 & 30 & 5 & 1 & 12 \\
\hline Number of orthologs in M. anisopliae & 6,863 & 6,705 & 158 & NA & NA \\
\hline Number of orthologs in M. acridum & 6,762 & 6,644 & NA & 118 & NA \\
\hline Number of orthologs in F. graminearum & 6,740 & 6,376 & 106 & 89 & 169 \\
\hline Number of orthologs in M. oryzae & 6,219 & 5,937 & 90 & 80 & 112 \\
\hline Percentage identity to $M$. anisopliae orthologs & 63.4 & 63.7 & 51.3 & NA & NA \\
\hline Percentage identity to $M$. acridum orthologs & 63.4 & 63.6 & NA & 51.2 & NA \\
\hline Percentage identity to $F$. graminearum orthologs & 61.6 & 62.3 & 51.9 & 52.8 & 46.7 \\
\hline Percentage identity to $M$. oryzae orthologs & 56.0 & 56.4 & 48.3 & 48.0 & 45.3 \\
\hline
\end{tabular}

${ }^{\mathrm{a}} \mathrm{CMM}$ core: $C$. militaris (CCM), M. anisopliae and M. acridum genes grouped with a cutoff $\mathrm{E}$ value of $1 \mathrm{e}-5$ during reciprocal Blast analysis. ${ }^{\mathrm{b}} \mathrm{CMA}$ restricted: $C$. militias and $M$. anisopliae restricted genes grouped with a cutoff $E$ value of $1 \mathrm{e}-5 .{ }^{c} \mathrm{CMC}$ restricted: $C$. militaris and $M$. acridum restricted genes grouped at a cutoff E value of $1 \mathrm{e}-5 .{ }^{\mathrm{d}} \mathrm{PHI}$ genes, pathogen-host interaction genes predicted by blast analysis against the PHI database [72]. Identity was estimated at the amino acid level. GPCR, G-protein coupled receptor; MFS, major facilitator superfamily; NA, not available; SM, secondary metabolite; TSA, trypsin, subtilisin and aspartyl protease.

cell walls - for example, GH6, GH7 and GH61 cellulases, GH10 and GH11 xylanases, GH28 pectinases and GH78 rhamnosidases (Table S7 in Additional file 1). There are also significant differences in the spectrum of enzymes produced by the entomopathogens. For example, compared to $M$. anisopliae, $C$. militaris has few xyloglucosyl transferases (GH16) for xyloglucan catabolism and lacks $\alpha$-glucuronidases (GH115) active on xylan oligomers or polymeric xylan [23]. Consistent with this, C. militaris grows very poorly on xylose when compared with M. anisopliae (Figure S2 in Additional file 2). A phosphoketolase MPK1 involved in pentose metabolism is required for full virulence of M. anisopliae [24], but the homolog is absent in C. militaris. However, GH18 chitinases similar to those used by Metarhizium to degrade insect cuticles [11] are well represented in the $C$. militaris genome (20 in CCM versus 30 in MAA and 19 in MAC) relative to plant pathogens (average 11) (Table S7 in Additional file 1).

Cytochrome P450s (CYPs) play essential roles in fungal physiologies, including detoxification, degradation of xenobiotics and the biosynthesis of secondary metabolites [25]. C. militaris has only about half as many CYPs as Metarhizium spp., and most other fungi (Table S8 in Additional file 1). Seventy CYP subfamilies present in M. anisopliae and/or M. acridum are absent in C. militaris. Of particular interest, C. militaris lacks CYP55, CYP58 and CYP65. CYP55 is a nitric oxide reductase required for denitrification [25]. Thus, unlike most filamentous fungi, $C$. militaris may not respond to hypoxia through the bacterial ammonia fermentation mechanism. The absence of CYP58 (trichodiene oxygenase) and CYP65 (trichothecene C-15 hydroxylase) suggests that C. militaris will not produce the mycotoxin trichothecene [26]. M. anisopliae can efficiently metabolize insect epicuticle alkanes [27]. The CYP52 subfamily for alkane hydroxylation [25] is well represented in Cordyceps.

The major facilitator superfamily (MFS) and ATPbinding cassette $(\mathrm{ABC})$ transporters are the two biggest families of fungal transporters. Members of the former typically function as nutrient symporters and drug antiporters, whereas the latter are more often implicated in 
defense against toxic metabolites [28]. C. militaris has approximately half (123) the number of these transporters as Metarhizium (269 in MAA and 236 in MAC) (Table S9 in Additional file 1). The MFS transporters that are underrepresented in Cordyceps include the carbohydrate symporters (37 in CCM versus 48 in MAA, 51 in MAC and an average of 58 in plant pathogens), vitamin B2 (riboflavin) transporters (2 in CCM versus 17 each in Metarhizium species and an average of 4 in plant pathogens) and multidrug antiporters (23 in CCM versus 110 in MAA, 77 in MAC and an average of 10 in plant pathogens). Consistent with their having many multidrug transporters, Metarhizium spp. are resistant to diverse antibiotics and fungicides [29]. Cordyceps has more $\mathrm{ABC}$-type drug and metal resistant proteins than Metarhizium and plant pathogens (63 in CCM, 56 in MAA, 51 in MAC and an average of 54 in plant pathogens). The amino acid and dipeptide transporters are similarly represented in the three insect pathogens and other fungi (46 in CCM versus 53 in MAA, 49 in MAC and an average of 45 in plant pathogens).

Fungal G-protein coupled receptors (GPCRs) are required for pheromone/nutrient sensing and host recognition [11]. Thus, the Pth11-like GPCR of Magnaporthe mediates cell differentiation in responses to plant inductive cues [30]. C. militaris has fewer GPCRs than Metarhizium spp. and is particularly impoverished in Pth11-like GPCRs (Table S10 in Additional file 1). C. militaris has a similar number (167) of protein kinases as M. anisopliae (161) but less than M. acridum (192) (Table S11 in Additional file 1). Like other fungi, fungal specific transcription factors (TFs) and zinc finger TFs represent the two largest classes of TFs in C. militaris and their numbers are similar to those of other fungi (Table S1 in Additional file 1).

\section{Mating-type and sexuality analysis}

The fruiting bodies of Cordyceps spp. are the most commonly sold traditional Chinese medicine products [5]. However, the sexual cycle and fruiting of $C$. militaris is poorly understood. We only identified a MAT1-1 mating-type locus, including MAT1-1-1 and MAT1-1-2 genes, in the sequenced $\mathrm{Cm} 01$ strain, suggesting that $C$. militaris is heterothallic (Figure 4a). A single matingtype locus was also found in M. anisopliae (MAT1-1) and M. acridum (MAT1-2). Like aspergilli [10], the idiomorphic regions of the three insect pathogens are highly divergent (Figure 4a). The MAT1-1 locus of M. anisopliae contains a MAT1-1-3 gene but lacks the MAT1-12 gene present in C. militaris. Except for the matingtype locus region, most $A$. nidulans and $N$. crassa genes involved in mating, fruiting, karyogamy and meiosis are also present in insect pathogens (Table S12 in Additional file 1).
Strain Cm01 forms fruiting bodies on caterpillar pupae that lack perithecia and ascospores (Figure 5a-e). Thus, it is the first ascomycete species reported to fruit without an opposite mating-type partner. Other C. militaris isolates could also fruit sterilely with a single mating-type locus (Figure 6a, b). However, a hybrid strain, Cm06, with both MAT1-1 and MAT1-2 loci produced sexual perithecia and ascospores (Figure 5f, g). In addition, the sexual structures could be similarly re-formed after inoculation of the caterpillar pupae with different ratios of MAT1-1 and MAT1-2 isolate conidia (Figure 6c-e), confirming that C. militaris is heterothallic. PCR examination of 18 field-collected strains identified three containing both MAT1-1 and MAT1-2 loci (Figure 5h). However, 28 out of 30 single spore isolates of the $\mathrm{Cm} 06$ strain belonged to the MAT1-1 mating-type (Figure 5i). A similar unequal prevalence of mating types occurs in the dermatophyte fungus [31].

\section{Metabolism of medically active components and mycotoxins}

One of the main pharmaceutically active components of C. militaris is cordycepin $[5,6]$, which is structurally similar to 2'-deoxyadenosine (Figure 7a). C. militaris possesses most of the genes required for metabolism of adenine and adenosine except for lacking a ribonucleotide trisphosphate reductase (RNR; converts ATP to dATP) and a deoxyadenosine kinase (converts deoxyadenosine to dAMP) (Figure 7b; Table S13 in Additional file 1). It has been suggested that the biosynthesis of cordycepin proceeds through a reductive mechanism as described for the formation of 2'-deoxyadenosine [32]. However, C. militaris resembles Metarhizium and other cordycepin non-producing fungi in having only two highly conserved subunits of class I RNRs (Figure S3 in Additional file 2). The substrates for class I RNRs are ADP, GDP, CDP and UDP but not TDP or nucleosides, and as the reductive reaction proceeds via a free radical mechanism [33], C. militaris RNRs will not be involved in cordycepin production.

Contamination of food and feed by mycotoxins is a longstanding threat to the health of humans and animals [26]. C. militaris has been consumed for hundreds of years, implying safety, but the genome data allowed us to make the first comprehensive inventory of Cordyceps genes involved in biosynthesis of secondary metabolites for comparison with known mycotoxins. There are fewer secondary metabolite core genes in $C$. militaris relative to Metarhizium spp. or plant pathogens (Table 3). In comparison to Metarhizium spp., Cordyceps has fewer terpenoid synthases, polyketide synthases (PKSs) and non-ribosomal peptide synthetases (NRPSs). Phylogenetic analysis of Cordyceps PKS and PKS-like genes using the ketoacyl CoA synthase (KS) domain sequences 


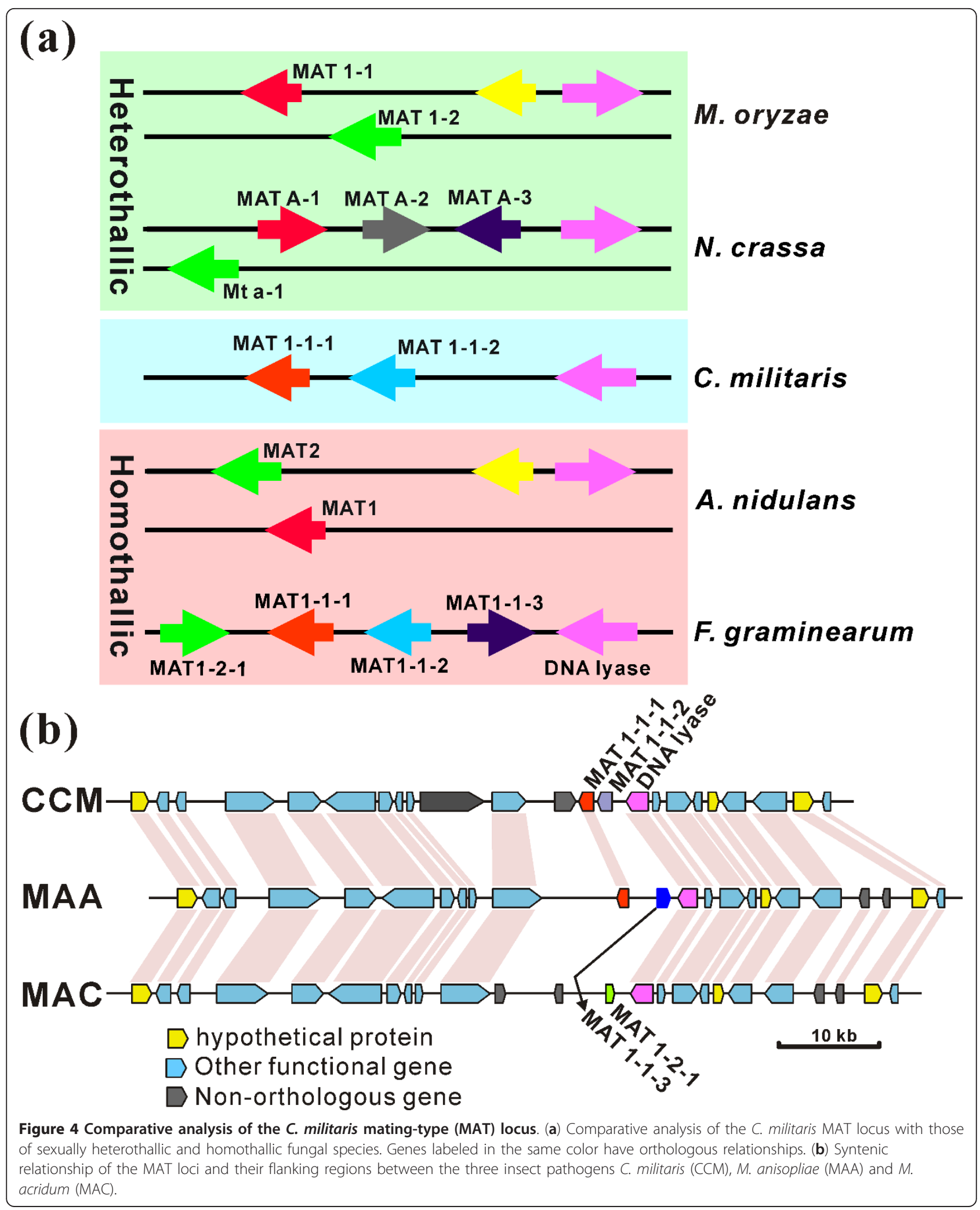




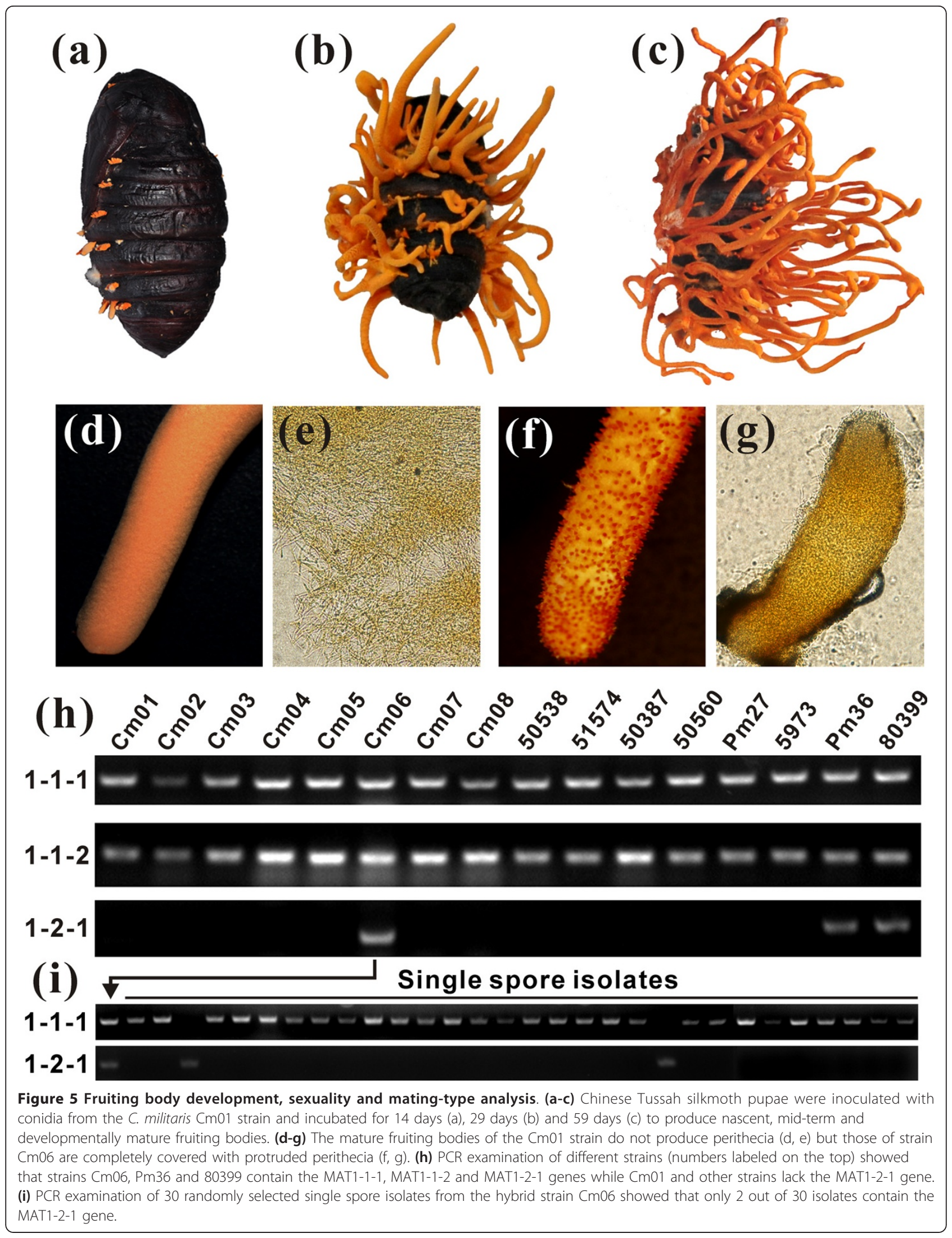



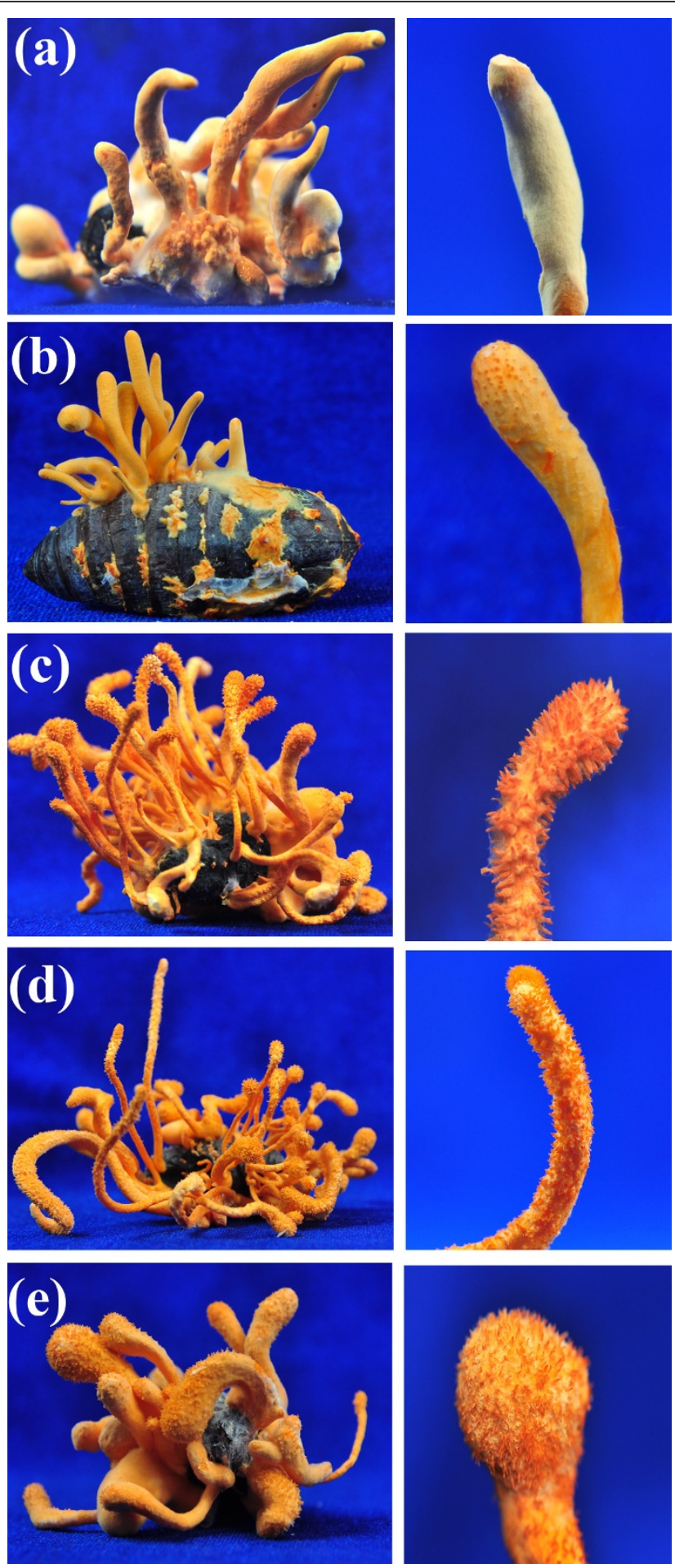

Figure 6 Fruiting structures of different mating-type isolates. (a, b) Sterile fruiting bodies formed on caterpillar pupae after inoculation of MAT1-1 (a) and MAT1-2 (b) isolates acquired by single conidial spore isolation from a MAT1-1/MAT1-2 hybrid strain, Cm06. (c-e) Fertile fruiting structures formed on caterpillar pupae after inoculation of the mixed conidia of MAT1-1 (Cm01) and MAT1-2 (Cm06) at ratios of 1:9 (c), 1:1 (d) and 9:1 (e), respectively. The right panels represent close-up views of corresponding sterile (without protruded perithecia) or fertile (with protruded perithecia) fruiting bodies. After inoculation, the pupae were incubated at $22^{\circ} \mathrm{C}$ with a $12: 12$ hour light:dark cycle for 60 days. 


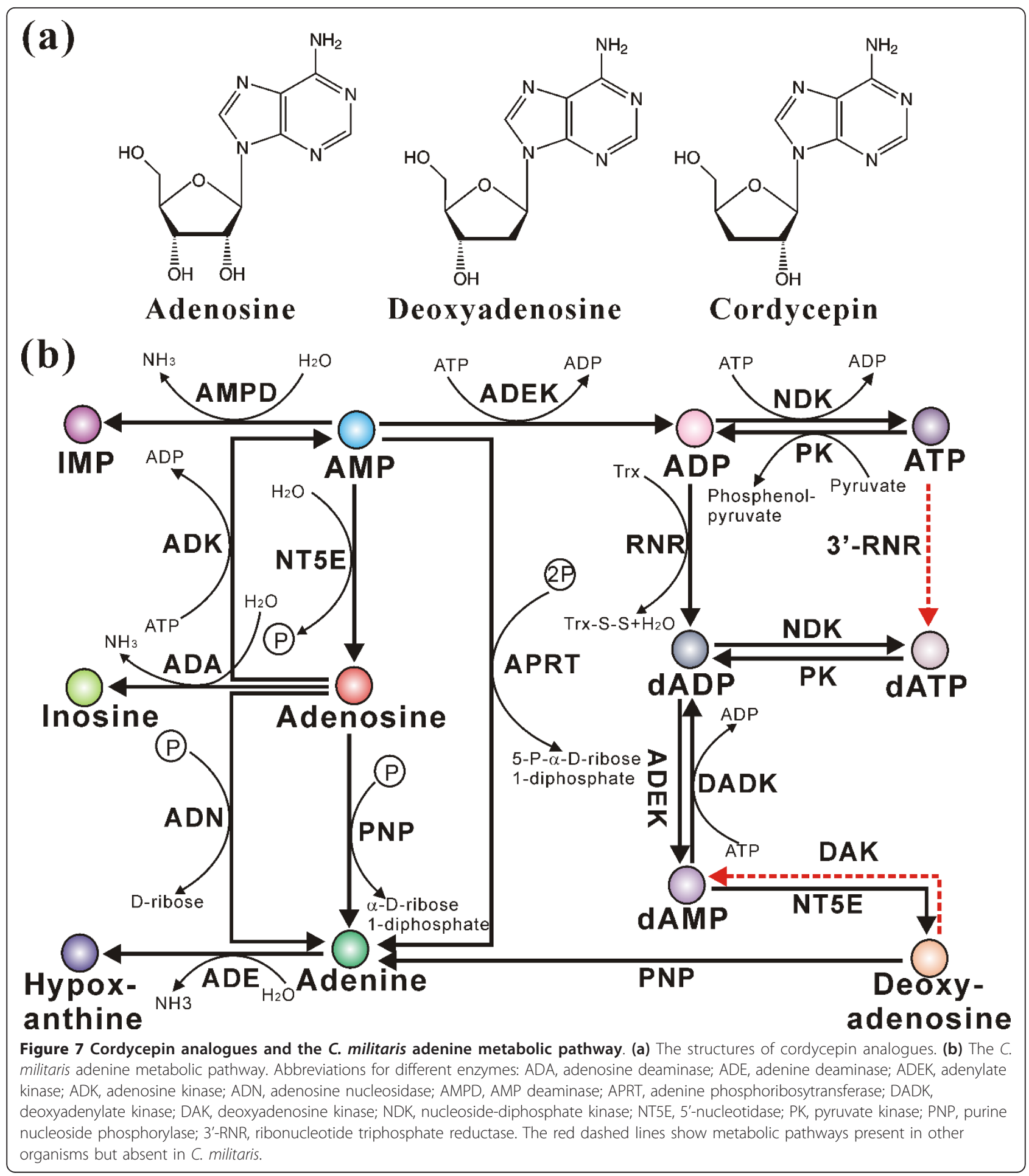

found that the $C$. militaris proteins grouped into different clusters compared to PKSs for known mycotoxins (Figure 8a). In addition, modular analysis indicated that, except for CCM_00603, which has a similar domain organization to the Aspergillus clavatus PatK gene for patulin biosynthesis, C. militaris PKSs are structurally different from mycotoxin PKSs (Figure 8b). The further survey showed that the CCM_00603 protein has only $27 \%$ identity with PatK and the gene cluster for patulin biosynthesis is absent in C. militaris (Table S14 in Additional file 1). This suggests that $C$. militaris PKSs do not produce patulin or other known human mycotoxins. 
Table 3 Numbers of core genes involved in the biosynthesis of secondary metabolites in different fungi

\begin{tabular}{lccccccccc}
\hline Core gene & CCM & MAA & MAC & FG & MO & BC & SS & NC & AN \\
\hline DMAT & 1 & 5 & 3 & 0 & 3 & 1 & 1 & 1 & 6 \\
TC & 3 & 3 & 3 & 3 & 3 & 3 & 3 & 3 & 5 \\
TS & 2 & 8 & 6 & 11 & 8 & 7 & 1 & 2 & 5 \\
FAS & 1 & 2 & 2 & 1 & 1 & 1 & 1 & 1 & 1 \\
GGPS & 3 & 4 & 4 & 3 & 3 & 0 & 0 & 1 & 0 \\
NRPS & 5 & 14 & 13 & 10 & 5 & 6 & 5 & 3 & 11 \\
NRPS-like & 8 & 9 & 8 & 11 & 6 & 8 & 5 & 3 & 12 \\
PKS & 9 & 24 & 13 & 14 & 12 & 16 & 16 & 6 & 24 \\
PKS-like & 2 & 3 & 4 & 1 & 3 & 6 & 2 & 2 & 4 \\
HYBRID & 3 & 5 & 1 & 1 & 3 & 0 & 0 & 0 & 1 \\
Total & 37 & 77 & 57 & 55 & 47 & 48 & 34 & 22 & 69 \\
\hline Core gen & & & & & & & &
\end{tabular}

Core genes encoding: DMAT, dimethylallyl tryptophan synthase; TC, terpenoid cyclase; TS, terpenoid synthase; FAS, fatty-acid synthase; GGPS, geranylgeranyl diphosphate synthase; NRPS, non-ribosomal peptide synthetase; PKS, polyketide synthetase; HYBRID, hybrid PKS-NRPS enzyme. Fungal species: CCM, C. militaris; MAA, M. anisopliae; MAC, M. acridum; FG, F. graminearum; $\mathrm{MO}, \mathrm{M}$. oryzae; $\mathrm{BC}$, Botrytis cinerea; SS, Sclerotinia sclerotiorum; NC, N. crassa; $\mathrm{AN}$, A. nidulans.

Similarly, phylogenetic and modular analyses indicated that Cordyceps NRPSs had different protein structures than any NRPSs involved in production of known mycotoxins like enniatin, HC-toxin and gliotoxin (Figure S4 in Additional file 2).

The mycotoxin ergot alkaloids have a wide range of biological activities and are important in pharmaceuticals and agriculture [26]. Dimethylallyl tryptophan synthase (DMAT) catalyzes the alkylation of L-tryptophan, the first committed step in the ergot alkaloid biosynthetic pathway [34]. C. militaris has one putative DMAT gene (CCM_04410), in contrast to five in $M$. anisopliae and three in M. acridum (Table 3). A phylogenetic analysis showed that CCM_04410 is not clustered with the Claviceps DMAT clade involved in ergot alkaloid production (Figure S5 in Additional file 2). The trichothecenes T-2 toxin and deoxynivalenol (type B trichothecene) are natural fungal products that are toxic to both animals and plants [35]. Consistent with lacking CYP58 and CYP65, the C. militaris genome also lacks trichodiene synthase (Table S15 in Additional file 1). Thus, unlike Fusarium [26], C. militaris is not predicted to produce trichothecene mycotoxins. The presence of terpenoid cyclase, terpenoid synthase, fatty-acid synthase and geranylgeranyl diphosphate synthase genes in the $C$. militaris genome suggests that the fungus is capable of producing an array of metabolites, but the identity of these and their biological activities remain to be determined.

Transcriptional regulation of fruiting body development To identify genes associated with C. militaris fruiting body development, we compared the expression profiles of undifferentiated mycelia from Sabouraud dextrose broth (SDB) culture with developmental stages on caterpillar pupae defined as nascent (14 days, termed as sample FB1), stalk formation (29 days, FB2) and mature fruiting bodies (59 days, FB3) (Figure 5a-c). Of the 9,684 genes, more than $63 \%$ were expressed during both undifferentiated hyphal growth and formation of fruiting bodies (Table S16 in Additional file 1). Relative to the growth in SDB, more than 900 genes were significantly $(P<0.05 ; \mathrm{FDR}<0.001)$ up-regulated while around 2,000 genes were down-regulated during fungal fruiting (Figure 9a). A Pearson correlation analysis indicated that transcriptional profiles at the different stages of fruiting body formation more closely resembled each other than they resembled the transcriptomes of undifferentiated mycelia (Figure S6a in Additional file 2). This is consistent with a Venn diagram analysis of the commonest co-expressed genes between different samples (Figure S6b in Additional file 2). Of the 100 most highly expressed genes in developing $C$. militaris fruiting bodies, 26 (FB1), 31 (FB2) and 37 (FB3) are functionally uncharacterized (Table S17 in Additional file 1). This suggests that the genes with unknown function are more likely to be stringently regulated and involved in developmental processes than orthologs of genes with known function. These genes are thereby the targets for future functional studies. In general, the genes involved in cell wall structure and biogenesis, detoxification, protein degradation and amino acid transportation were significantly up-regulated during formation of fruiting structures. In contrast, most of the genes specifically upregulated by undifferentiated SDB cultures were involved in rapid growth and carbohydrate metabolism. Concomitant with fruiting structure maturation, the genes for cytoskeletal organization, cell cycle and secondary metabolism were up-regulated.

Unlike other fungi, C. militaris can fruit sterilely in the absence of a sexual partner (Figure 5a-c). Perhaps because of this, 31 of the 42 C. militaris orthologs of sex-related genes identified in other ascomycetes were not expressed or transcribed at low levels $(<10$ transcripts per million tags (TPM)) in sterile fruiting bodies (Table S18 in Additional file 1). However, in some cases, $C$. militaris expresses paralogous genes to those employed by other fungi, suggesting they have co-opted different components of the same signal transduction pathways to fulfill similar functions. For example, GATA-type TFs are important for fruiting in both $A$. nidulans and $N$. crassa [36], but C. militaris fruiting structures expressed orthologs of these genes at very low levels or not at all (Table S19 in Additional file 1). In contrast, the Zn2Cys6-type TFs were highly transcribed during fruiting but not in undifferentiated fungal mycelia - for example, CCM_01809 and CCM_09644 


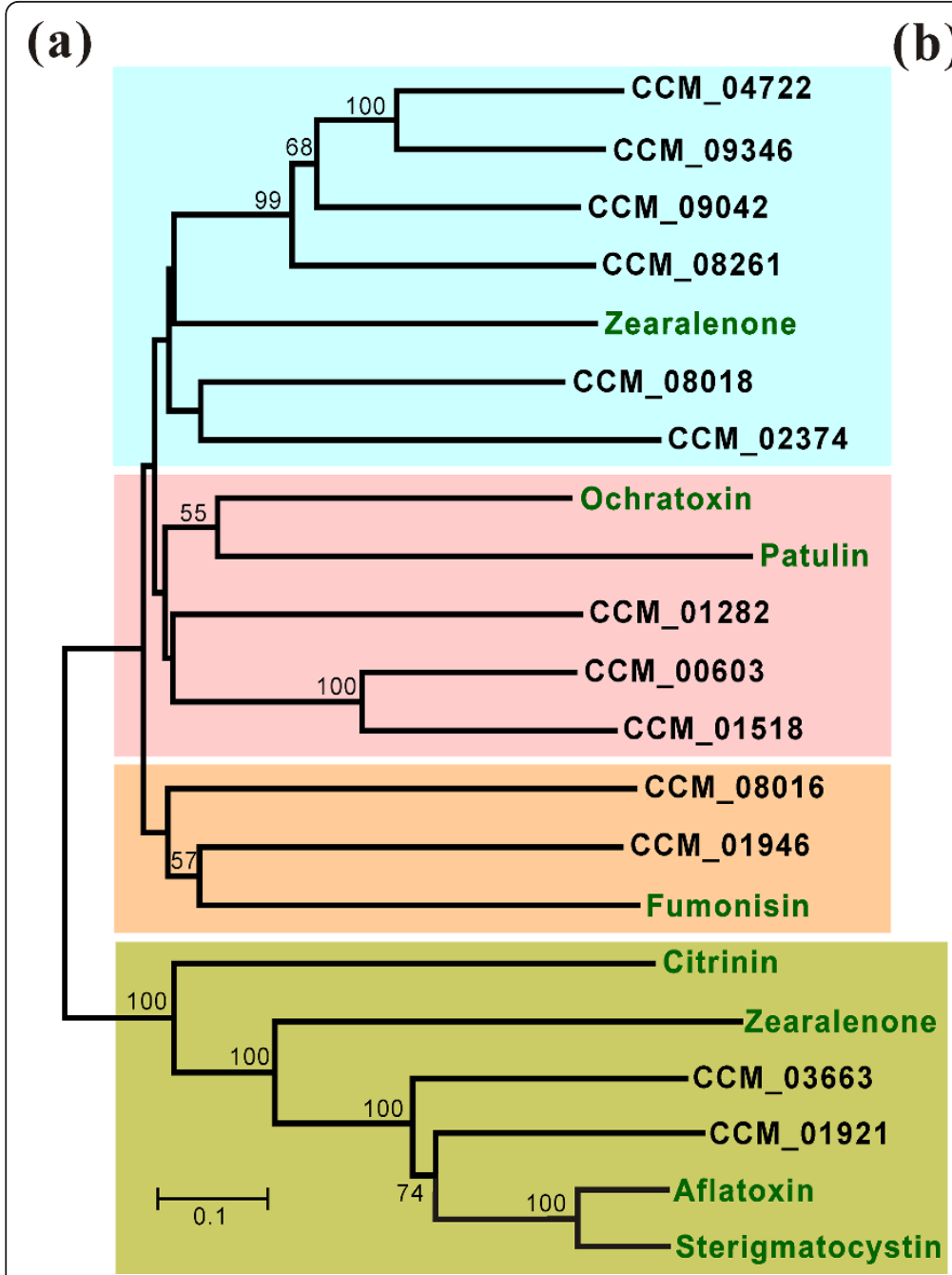

(b)

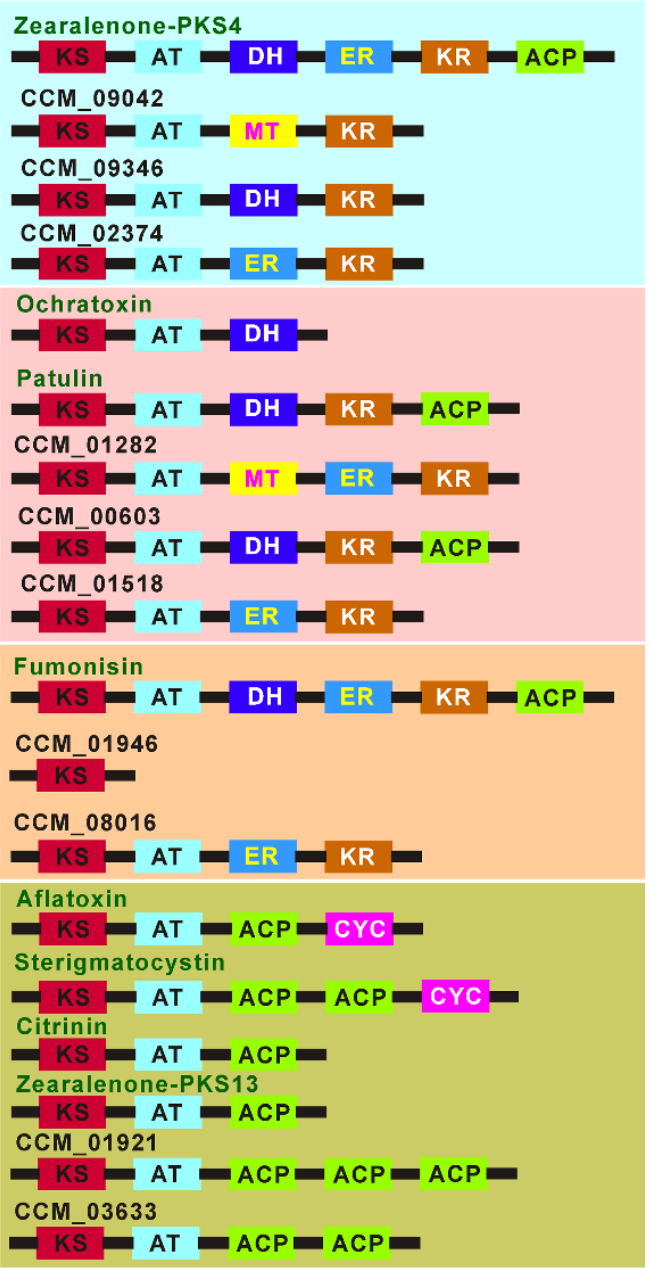

Figure 8 Phylogenetic and modular analysis of $C$. militaris polyketide synthases compared with those involved in the production of human mycotoxins. (a) A neighbor-joining tree showing the relationships of ketoacyl CoA synthase (KS) domain sequences. (b) Modulation and comparison of C. militaris PKSs with those involved in production of mycotoxins. The PKS-NRPS hybrid proteins CCM_04722, CCM_08261 and CCM_08018 are not included in the analysis. Domain definitions: ACP, acyl carrier protein domain; AT, acyltransferase domain; CYC, Cyclase domain; DH, dehydratase domain; ER, enoyl reductase domain; KR, ketoreductase domain; MT, methyltransferase domain; TE, thioesterase domain. The accessions and references for different mycotoxins are provided in the Materials and methods.

(Figure 9b) - indicating that Zn2Cys6 type TFs are predominately involved in the major developmental switch of production of fruiting structures.

Pheromone receptors, that is, GPCRs, control fungal fruiting body formation and sexual cycle but not vegetative growth [36]. The pheromone receptor of C. militaris has not been identified. In comparison to undifferentiated mycelial growth, a putative pheromone receptor (CCM_01499) and a Pth11-like GPCR (CCM_03015) were significantly up-regulated $(P<0.05$, FDR $<0.001$ ), respectively, during initiation of fruiting body formation. Mitogen-activated protein kinase (MAPK) genes are required for fruiting in Aspergillus (AN1017) and Neurospora (NC02393) [36], but orthologous genes were not transcribed (CCM_04200 versus AN1017) or transcribed at low levels (CCM_01235 versus NCU02393) by C. militaris (Table S19 in Additional file 1). However, Cordyceps sharply up-regulated $(P<0.05$, FDR $<0.001)$ a MAPK paralog (CCM_09637) as well as a calcium regulated kinase (CaMK, CCM_06085) (Figure 9c). These data, taken in conjunction with the single adenylate cyclase (CCM_06928) not being transcribed and the low level expression of both protein kinase A (PKA; CCM_03352) and Rap GTPase (CCM_01391), indicate that fruiting by C. militaris in the absence of a partner is more dependent on the MAPK pathway than the cAMP-dependent PKA pathway (Figure 10). 


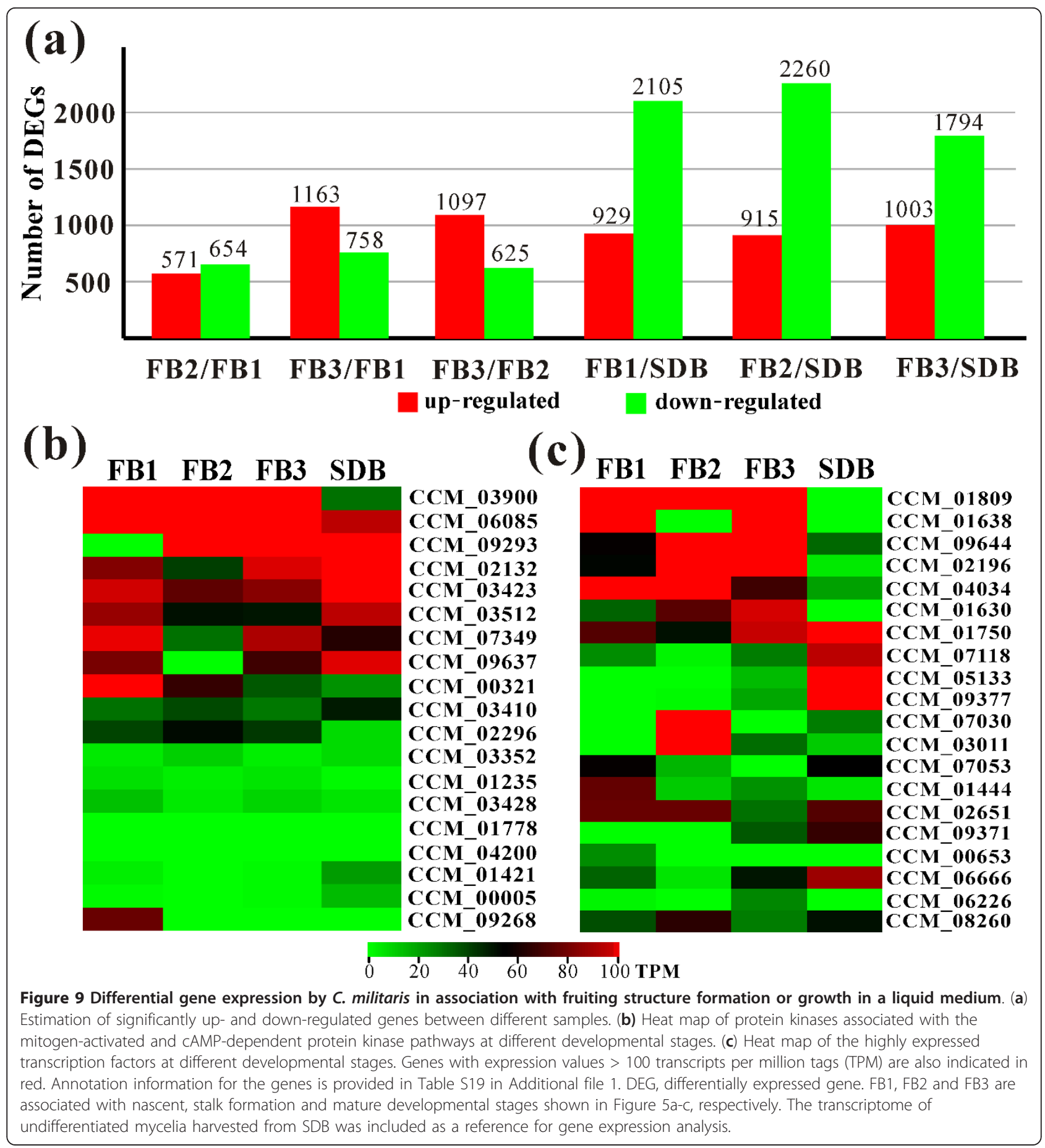

\section{Discussion}

We report here the first genome analysis of a Cordyceps species, the medicinal lepidopteran pathogen C. militaris, and show that the fungus is capable of fruiting without an opposite mating-type partner. We also show that it lacks genes known to be involved in production of human mycotoxins. Being an insect pathogen, the $C$. militaris genome contains thousands of genes putatively involved in interactions with insect hosts. Cordyceps resembles Metarhizium spp. in having a very high percentage of secreted proteins relative to plant pathogens and saprophytes and expanded families of proteases and chitinases with targets in insect hosts. However, insectkilling strategies may differ between Cordyceps and Metarhizium due to differences in gene content. Mating-type analysis indicated that sexual reproduction in 


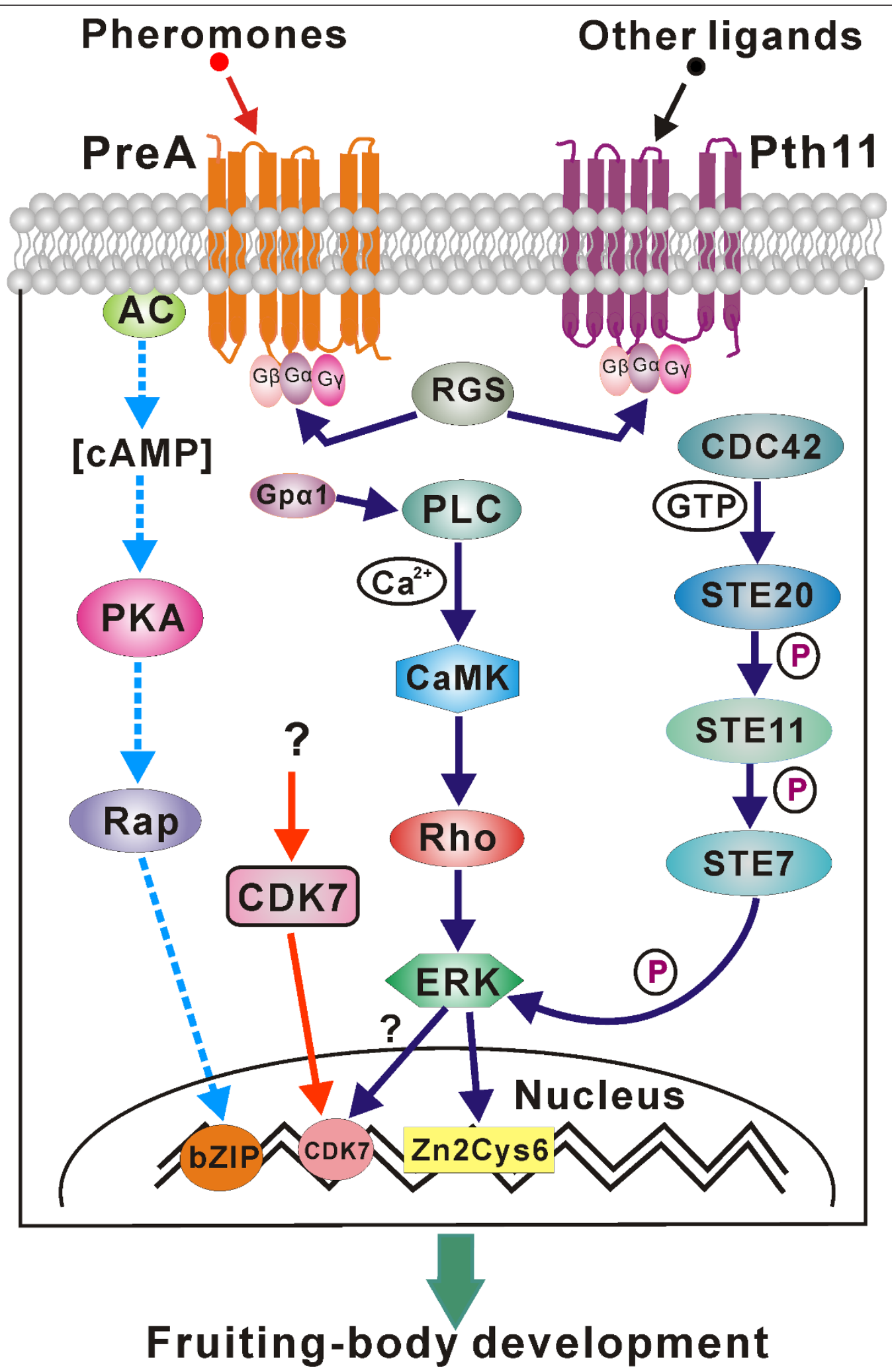

Figure 10 Putative signal transduction pathways regulating fruiting body development in C. militaris. The dashed lines show the CAMPdependent PKA pathway, which might not be involved in control of fruiting in C. militaris. The transcription data for different components are provided in Table S19 in Additional file 1. AC, adenylate cyclase; CaMK, calmodulin-dependent protein kinase; CDK, cyclin-dependent kinase; PLC, phospholiapse C; RGS, regulator of G protein signaling. 
C. militaris is heterothallic. Transcriptional profiling indicated that fruiting of the MAT1-1 C. militaris strain involves induction of the MAPK pathway, but unlike other homothallic or heterothallic fungi, the PKA pathway was not up-regulated. It remains to be determined whether this reflects the very unusual ability of $C$. militaris to produce fruiting bodies without an opposite mating-type partner.

Aside from knowing that $C$. militaris infects lepidopteran pupae [37], the life cycle of $C$. militaris in nature is poorly understood [8]. Following disease, survival in soil may depend on the sexual stage of Cordyceps providing resilient long-lived ascospores as described in other fungi [38]. Micro-cycle conidiation from germinated ascospores could adapt the fungus to nutrient poor niches (Figure 1). Metarhizium does not produce ascospores but flourishes in plant rhizospheres, which thus provide an alternative habitat in the absence of insect hosts. C. militaris can grow on germinated soybeans [39], suggesting a potential for an association with plants. However, relative to Metarhizium and most other ascomycetes, many protein families are smaller in the $C$. militaris genome, especially serine proteases, GHs, CYPs, MFS transporters and signal transduction factors. These families would be involved in scavenging for nutrients, avoidance of host defenses and toxins and other processes related to pathogenicity and a saprobic lifestyle. Around two-thirds of these protein families include pathogen-host interaction genes in plant-associated fungi (Table S4 in Additional file 1). Further studies on the ecology of Cordyceps spp. will shed more light on the relevance of the $C$. militaris genome to the evolution of gene families in relation to acquisition/loss of capability for dual plant/insect colonization and host range specialization.

The phylogenomic analysis demonstrated that the lineage leading to Cordyceps spp. diverged after most well known plant pathogens, including F. graminearum, but 130 MYA before Metarhizium diverged from the grass endophyte Epichloë festucae. The estimate of a Triassic-Jurassic boundary origin for the Cordyceps lineage and the post-Cretaceous origin of Metarhizium spp. is consistent with the hypocrealean fungi of Cordycipitaceae (includes Cordyceps spp.), Clavicipitaceae (includes Metarhizium spp.) and Ophiocordycipitaceae splitting about the same time as insects and angiosperms were diversifying [40]. Families of proteases and chitinases are not expanded or lost in the E. festucae genome as they are in Cordyceps and Metarhizium, exemplifying convergent evolution to insect pathogenicity. Besides proteases and chitinases, experimentally verified Metarhizium virulence-associated genes with homologs in the C. militaris genome include a perilipin-like protein (CCM_06103 versus MAA_08819) to control cellular lipid storage and appressorium penetration [41], and an osmosensor (CCM_04885 versus MAA_01551) to mediate adaptation to the insect hemocoel [42]. Homologs of these genes are broadly distributed among ascomycetes, indicative of an ancient origin. However, Cordyceps lacks other Metarhizium pathogenicity-related genes, including a collagen-like protein to evade the host immune system [43], a phosphoketolase for pentose metabolism [24] and the adhesins to mediate spore adhesions to insect and plant surfaces [44]. The absence of key components of the Metarhizium entomopathogenicity 'toolkit' from $C$. militaris indicates that it has evolved different determinants to mediate its interactions with insects.

Like $N$. crassa and F. graminearum, C. miltaris lacks highly similar paralogs, a hallmark of the repeat-induced point mutation (RIP) mechanism [20]. C. militaris has an ortholog (CCM_03609) of the $N$. crassa RIP defective gene (NCU02034), a cytosine methyltranserase essential for RIP [45]. The high $\mathrm{C} \rightarrow \mathrm{T}$ and $\mathrm{G} \rightarrow \mathrm{A}$ mutation bias in the $C$. militaris genome and the readiness of $C$. miltaris to undergo the sexual cycle suggests that RIP is commonplace in $C$. militaris like many ascomycetes $[10,15,19]$. Since RIP can function effectively against selfish DNAs [46], it likely contributes, at least in part, to $C$. militaris having few DNA type transposon encoded genes, that is, transposases [15].

There are many more orphan genes in Cordyceps than in Metarhizium spp., underscoring that much about the proteome of Cordyceps spp. remains unknown. It is speculated that orphan genes arise from gene duplication, shuffling of gene fragments, mobile element effects, mutation of existing sequences, horizontal gene transfer and de novo origination from non-coding DNAs [47]. De novo creation of new genes is probably rare [48]. A role for mobile element effects is also unlikely given how few putative transposase genes are present in the $C$. militaris genome. Putative horizontal gene transfer genes are even fewer in Cordyceps than in Metarhizium. Thus, the numerous orphans in the $C$. militaris genome most likely arose from frequent mutations caused by RIP in existing (duplicated) sequences. Just as the Metarhizium-specific collagen-like protein is essentially required to camouflage cells from host immune recognition [43]. The transcriptome data showed that 428 of the 1,329 orphan genes were transcribed during fruiting. Of the 100 most highly expressed genes in developing $C$. militaris fruiting bodies, about one-third are orphans (Table S17 in Additional file 1), underscoring the potential of orphans to have specific functions. Likewise, genes that are apparently unique to the mushroom Schizophyllum commune are more likely to be expressed during mushroom formation [49]. 
Concern has been raised about the possibility of harmful side effects of traditional Chinese medicines, including Cordyceps [50]. Consistent with genotoxicity and cytotoxicity assays that show Cordyceps products to be safe for consumption [51], there is no evidence in the C. militaris genome for genes involved in the production of known mycotoxins. However, safety could only be completely verified by meticulous profiling of the metabolites produced by the fungus under diverse growth conditions. The $C$. militaris genome data will facilitate these processes as well as help with elucidation of the biosynthetic pathways of different metabolites.

The analysis of $C$. militaris genome indicates that it is sexually heterothallic, but strikingly, both the MAT1-1 single mating-type and MAT1-1/MAT1-2 hybrid strains can form fruiting bodies, which means that $C$. militaris is capable of fruiting without a partner. Single matingtype (haploid) fruiting has also been observed in the human pathogens Cryptococcus neoformans and Candida albicans [52]. Given that perithecia and ascospores are not produced by MAT1-1 fruiting bodies, C. militaris haploid fruiting is different from the same-sex mating and fruiting of $C$. neoformans, in which diploidization and meiosis can occur. In budding yeast, meiotic recombination is initiated by the formation of double-strand breaks catalyzed by SPO11, a meiosis-specific endonuclease [53]. The meiosis-specific recombinase DMC1 and the DNA repair enzyme RAD51 then co-localize to double-strand breaks and function together for meiotic recombination [54]. The C. militaris homologue of yeast SPO11 (CCM_09527) was upregulated more than five-fold during fruiting body maturation (the TPM ratio of $\mathrm{FB} 2 / \mathrm{FB} 1=8.1$; FB3/FB2 = 5.7). Intriguingly, the $C$. militaris genome lacks a yeast RAD51 ortholog, but its DMC1 ortholog (CCM_06822) contains a RAD51 domain. CCM_06822 was not expressed by $C$. militaris during fruiting, which may explain why the $C$. militaris MAT1-1 strain forms fruiting bodies without meiosis. Consistent with this, a putative cyclin dependent kinase 7 (CDK7; CCM_03900) was up-regulated during fungal fruiting (Table S19 in Additional file 1). Orthologs of CDK7 initiate DNA synthesis and facilitate mitosis instead of meiosis [55].

\section{Conclusions}

In conclusion, we report on the genome sequencing, comparative genome analysis and transcriptional regulation of fruiting body development in the medicinal fungus $C$. militaris. The sequence data should markedly enhance the pace of molecular research on Cordyceps biology, fungal sex and pathogenicity, and will have impacts on the commercial production of fruiting structures. The genomic sequence will also be an essential tool to unravel the mechanisms by which $C$. militaris produces medicinal compounds and so further their exploitation.

\section{Materials and methods \\ Fungal strains}

C. militaris strain Cm01 (CGMCC 3.14242) was selected for genome sequencing as it is culturally stable and commercialized in China. The culture was maintained either on artificial medium or silkworm pupae as previously described [12]. Several different C. militaris strains were included in this study for PCR genotyping of mating-type genes (Figure 5h).

\section{Genome sequencing and assembly}

The genome of $C$. militaris strain $\mathrm{Cm} 01$ was shotgun sequenced using a Roche 454 GS FLX system for massively parallel pyrosequencing for 2.25 runs at the Chinese National Human Genome Center (Shanghai, China). This resulted in $951 \mathrm{Mb}$ of sequence data (29.6 $\times$ coverage) with an average read length of $385 \mathrm{bp}$. Assembly was performed using the Newbler software (v2.3) within the Roche 454 suite package [56], which produced 597 contigs with a total size of $32.2 \mathrm{Mb}$. For sequence scaffolding, a DNA library of 2- to 5-kb inserts was generated and sequenced with an ABI SOLiD system (Carlsbad, California, USA). This resulted in $3.8 \mathrm{~Gb}$ of mate-pair reads $(117.4 \times$ coverage $)$ to improve sequence quality and construct scaffolds. By mapping the reads to contigs, 578 contigs were assembled into 13 scaffolds and 19 contigs less than $2 \mathrm{~kb}$ left outside. The raw data of 454 and SOLiD reads have been deposited at NCBI's Sequence Read Archive under accession number SRA047932 and the whole project has been deposited at DDBJ/EMBL/GenBank under accession number AEVU00000000.

\section{Annotation}

To maximize gene prediction accuracy, the gene structures of Cordyceps were predicted with a combination of different algorithms plus manual inspections [11,57]. The inconsistent open reading frames were individually subject to Blast searches against the NCBI curated refseq_protein database. The prediction with the best hit was selected. Pseudogene identification was conducted with the pipeline of PseudoPipe with default settings [58]. The potential secreted proteins of $C$. militaris and other fungal species included for comparison were predicted by SignaIP 3.0 analysis using a hidden Markov model [59]. Genome repetitive elements were analyzed by Blast against the RepeatMasker library (Open 3.2.9) [60] and with the Tandem Repeats Finder [61]. The transposases/retrotransposases were classified by Blastp analysis against the Repbase [62] plus manual inspections. 


\section{Blast score ratio test}

BSR tests [14] were conducted to compare the differences between $C$. militaris and the sequenced Metarhizum genomes and the plant pathogen F. graminearum, respectively. The BSR index for each reference protein is calculated by dividing the query bit score by the reference score and normalized from 0 to 1 . A score of 1 indicates a perfect match while a score of 0 indicates no Blast match of a query protein in the reference proteome. The normalized pairs of BSR indices were then plotted using the Matlab (v7.0) program (Natick, Massachusetts, USA). The same analysis was conducted for the three genomes of $C$. militaris, M. anisopliae and $F$. graminearum.

\section{Orthology and phylogenomic analysis}

In total, 2,106 orthologous proteins were acquired by a reciprocal Blast method with a cutoff $E$ value of $1 \mathrm{e}-20$ and a Blast alignment length greater than $60 \%$ of the query sequence. Corresponding orthologous gene protein sequences were aligned with Clustal X 2.0 and the concatenated amino acid sequences were used for the generation of a maximum likelihood phylogenomic tree with the program TREE-PUZZLE [63] using a Dayhoff model. The divergence time between species was estimated with the program r8s using a Langley-Fitch model [64] by calibration with the origin of the Ascomycota at 500 to 650 MYA [65].

\section{Protein family classifications}

Whole genome protein families were classified by InterproScan [66] and Pfam [67] analysis. The families of proteases were identified by Blastp searching against the MEROPS peptidase database release 9.4 with a cutoff $\mathrm{E}$ value of $1 \mathrm{e}-20$ [68]. The CYPs were named according to the classifications collected at the P450 database [69]. Transporters were classified based on the Transport Classification Database [70]. Kinases were classified by Blastp analysis against the KinBase database with a cutoff $E$ value of 1e-10 [68]. Carbohydrate-active enzymes were classified by local Blastp searching against a library of catalytic and carbohydrate-binding module enzymes [68]. G-protein-coupled receptors were selected from the best hits to GPCRDB sequences [71] and by confirmation that they contained seven transmembrane helices with the amino terminus outside and the carboxyl terminus inside the plasma membrane. Homologs of the Magnaporthe Pth11-like GPCRs [30] were identified by local Blastp analysis with a cutoff $E$ value of $1 \mathrm{e}-$ 10. Putative Cordyceps virulence factors were identified by searching against the pathogen-host interaction database [72] with a cutoff $E$ value of $1 \mathrm{e}-5$, plus additional searches of known virulence genes reported in entomopathogenic fungi. Two sample $t$-tests were conducted to compare the differences in protein family sizes between insect and plant pathogens. Estimation of FDR of $P$ values was conducted using the program mafdr (Matlab 7.8.0.347(R2009a)).

\section{Analysis of genes involved in purine synthesis and secondary metabolism}

To model the biosynthesis of cordycepin, the purine metabolic pathway in C. militaris was constructed based on the KEGG (Kyoto Encyclopedia of Genes and Genomes) annotations [73]. To identify NRPS, PKS or NRPS-PKS hybrid genes and gene clusters, the whole genome data set was subjected to analysis with the program SMURF with default settings [74]. Modulation analysis and domain extraction of different NRPS or PKS proteins were conducted by Blast searching against the SBSPKS database [75]. For phylogenetic analysis, the domain sequences were aligned with Clustal X 2.0 and the tree was generated using a Poisson model with 1,000 bootstrap replications and pair-wise deletions for gaps or missing data. The mycotoxin-encoding PKSs used in the analysis include Gibberella zeae PKS4 (ABB90283) and PKS13 (ABB90282) for the biosynthesis of zearalenones [76], Aspergillus ochraceus PKS (AAT92023) for ochratoxin [77], A. clavatus PatK (ACLA_093660) for patulin [78], Gibberella moniliformis Fum1p (AAD43562) for fumonisin [79], Monascus purpureus PKS (BAD44749) for citrinin [80], Aspergillus flavus PksA (AAS90093) for aflatoxin [81] and A. nidulans StcA (Q12397) for sterigmatocystin [82]. The mycotoxin-encoding NRPSs included in the analysis are $A$. fumigatus Glip (AAW03307) for gliotoxin [83], Fusarium equiseti NRPS (CAA79245) for enniatin [84], Cochliobolus carbonum NRPS (AAA33023) for HCtoxin [85] and Tolypocladium inflatum NRPS (CAA82227) for cyclosporin [86].

\section{Transcriptome analysis}

Conidia of $C$. militaris from day 14 potato dextrose agar were inoculated into SDB and the undifferentiated mycelia harvested after a 72 -hour incubation at $25^{\circ} \mathrm{C}$, $180 \mathrm{rpm}$. The transcriptome of the mycelia provided a control for comparison with the transcriptomes of fruiting bodies. Chinese Tussah silkmoth (Antheraea pernyi) pupae were injected with $50 \mu \mathrm{l}$ of a conidial suspension $\left(5 \times 10^{6}\right.$ conidia/ml $)$ and incubated at $22^{\circ} \mathrm{C}$ in a $12: 12$ hour light:dark cycle for up to 14 days to allow emergence of fruiting bodies (a stage designated as FB1), 29 days for half-grown fruiting bodies (FB2) and 59 days, by which time fruiting bodies were mature (FB3) $[11,12]$. RNA was extracted with a Qiagen RNeasy kit plus on-column treatment with RNase-free DNase I (Germantown, Maryland, USA). Messenger RNA was purified and after reverse transcription into cDNA, the 
libraries were constructed for tag preparation according to the massively parallel signature sequencing protocol [87]. The tags were sequenced with an Illumina technique. We omitted tags from further analysis if only one copy was detected or it could be mapped to a different transcript. Other tags were mapped to the genome or annotated genes if they possessed no more than one nucleotide mismatch $[11,88]$. The abundance of each tag was converted to the value of transcripts per million (TPM) for each mapped gene for expressional comparison between samples. The significance of differential gene expression between samples and the FDR of $P$ values were estimated for each individual gene with a cutoff of $P \leq 0.05$ and FDR $\leq 0.001[11,89]$. The RNA_seq expression dataset is available at the NCBI's Gene Expression Omnibus under the accession code GSE28001.

\section{Additional material}

Additional file 1: Comparative genomics analysis of $\boldsymbol{C}$. militaris. The file contains additional information on genomic properties and comparative gene family analysis of $C$. militaris with other fungi comprising 19 tables provided in separate excel sheets. Table S1 summarizes major protein family sizes of different fungal species. Table S2 provides a comparison of transposase genes among three insect pathogens. Table S3 lists the pseudogenes present in the genomes of three insect pathogens. Table \$4 summarizes the protein families putatively involved in pathogen-host interactions. Table S5 compares the proteases in different fungal genomes. Table $\mathbf{S} 6$ lists the serine and aspartyl proteases in three insect pathogens. Table S7 lists the glycoside hydrolase families in different fungal genomes. Table S8 compares the cytochrome P450 genes in three insect pathogens. Table S9 summarizes the membrane transporters in different fungal genomes. Table S10 compares the G-protein-coupled receptors in three insect pathogens. Table S11 lists the protein kinases in three insect pathogens. Table S12 provides the information of mating- and sexuality-related genes. Table S13 lists the genes putatively involved in purine metabolisms in three insect pathogens. Table S14 summarizes the presence/absence of patulin biosynthesis homologous genes in C. militaris. Table S15 summarizes the presence/absence of T-2 toxin biosynthesis homologous genes in C. militaris. Table S16 summarizes the information from RNAseq analysis. Table S17 lists the 100 most highly expressed genes in C. militaris at different growth stages. Table S18 lists the transcriptional data of sexuality- and fruiting-related genes. Table S19 compares the expression data of genes putatively involved in signaling and transcription controls.

Additional file 2: Figures that provide support information for the main text. Figure S1 provides support for RIP occurring in C. militaris. Figure S2 provides support for the lack of the pentose metabolic pathway in C. militaris. Figure S3 provides a phylogeny analysis of fungal ribonucleotide reductases. Figure S4 provides the phylogeny and modular analysis of C. militaris NRPSs. Figure S5 provides a phylogeny analysis of fungal dimethylallyl tryptophan synthases. Figure $\mathbf{S 6}$ provides the gene transcription profiles between different samples.

\section{Abbreviations}

ABC: ATP-binding cassette; bp: base pair; BSR: Blast score ratio; CCM: Cordyceps militaris; CYP: cytochrome P450; DMAT: dimethylallyl tryptophan synthase; FDR: false discovery rate; GH: glycoside hydrolase; Gl: genomic island; GPCR: G-protein coupled receptor; MAA: Metarhizium anisopliae; MAC: Metarhizium acridum; MAPK: mitogen-activated protein kinase; MAT: mattingtype; MFS: major facilitator superfamily; MYA: million years ago; NRPS: non- ribosomal peptide synthetase; PKA: protein kinase A; PKS: polyketide synthase; RIP: repeat-induced point mutation; RNR: ribonucleotide trisphosphate reductase; SDB: Sabouraud dextrose broth; TF: transcription factor; TPM: transcripts per million tags.

\section{Acknowledgements}

CSW was supported by the Ministry of Science and Technology of China (grant number 2009CB118904), the Ministry of Agriculture of China (grant number 2009ZX08009-035B), the Science and Technology Commission of Shanghai Municipality (grant number 08DZ1970200) and the Chinese Academy of Sciences (KSCX2-EW-N-06 and KSCX2-EW-G-16).

\section{Author details}

${ }^{1}$ Key Laboratory of Insect Developmental and Evolutionary Biology, Institute of Plant Physiology and Ecology, Shanghai Institutes for Biological Sciences, Chinese Academy of Sciences, 300 Fenglin Road, Shanghai 200032, China. ${ }^{2}$ Chinese National Human Genome Center at Shanghai, 250 Bibo Road, Shanghai 201203, China. ${ }^{3}$ Institute of Microbiology, Chinese Academy of Sciences, 1 West Beichen Road, Beijing 100101, China. ${ }^{4}$ Department of Entomology, University of Maryland, 4112 Plant Sciences Building, College Park, Maryland 20742, USA.

\section{Authors' contributions}

CSW initiated and designed the study. PZ, YLX, GHX, CHX, YH and YZ annotated genes and performed protein family analysis; GHX and HX performed phylogenetic and transcriptome analysis; PZ and SWZ conducted fruiting body induction, and DNA and RNA extraction; HJZ, SYW and GPZ performed genome sequencing and assembly; PZ and GHX performed transcriptome analysis; CSW and RJSL wrote the paper. All authors read and approved the final manuscript.

Received: 4 July 2011 Revised: 10 November 2011

Accepted: 23 November 2011 Published: 23 November 2011

\section{References}

1. Sung GH, Hywel-Jones NL, Sung JM, Luangsa-Ard JJ, Shrestha B, Spatafora JW: Phylogenetic classification of Cordyceps and the clavicipitaceous fungi. Stud Mycol 2007, 57:5-59.

2. de Faria MR, Wraight SP: Mycoinsecticides and Mycoacaricides: a comprehensive list with worldwide coverage and international classification of formulation types. Biol Control 2007, 43:237-256.

3. St Leger RJ, Wang C: Genetic engineering of fungal biocontrol agents to achieve greater efficacy against insect pests. Appl Microbiol Biotechnol 2010, 85:901-907.

4. Paterson RR: Cordyceps: a traditional Chinese medicine and another fungal therapeutic biofactory? Phytochemistry 2008, 69:1469-1495.

5. Zhou X, Gong Z, Su Y, Lin J, Tang K: Cordyceps fungi: natural products, pharmacological functions and developmental products. J Pharm Pharmacol 2009, 61:279-291.

6. Xiao JH, Zhong Jj: Secondary metabolites from Cordyceps species and their antitumor activity studies. Recent Pat Biotechnol 2007, 1:123-137.

7. ClinicalTrials.gov.. [http://clinicaltrials.gov/show/NCT00709215].

8. Stone R: Last stand for the body snatcher of the Himalayas? Science 2008, 322:1182.

9. Lee SC, Ni M, Li W, Shertz C, Heitman J: The evolution of sex: a perspective from the fungal kingdom. Microbiol Mol Biol Rev 2010, 74:298-340.

10. Galagan JE, Calvo SE, Cuomo C, Ma LJ, Wortman JR, Batzoglou S, Lee SI, Bastürkmen M, Spevak CC, Clutterbuck J, Kapitonov V, Jurka J, Scazzocchio C, Farman M, Butler J, Purcell S, Harris S, Braus GH, Draht O, Busch S, D'Enfert C, Bouchier C, Goldman GH, Bell-Pedersen D, GriffithsJones S, Doonan JH, Yu J, Vienken K, Pain A, Freitag M, et al: Sequencing of Aspergillus nidulans and comparative analysis with $A$. fumigatus and $A$. oryzae. Nature 2005, 438:1105-1115.

11. Gao Q, Jin K, Ying SH, Zhang $Y$, Xiao G, Shang $Y$, Duan $Z$, Hu X, Xie XQ, Zhou G, Peng G, Luo Z, Huang W, Wang B, Fang W, Wang S, Zhong Y, Ma LJ, St Leger RJ, Zhao GP, Pei Y, Feng MG, Xia Y, Wang CS: Genome sequencing and comparative transcriptomics of the model entomopathogenic fungi Metarhizium anisopliae and M. acridum. PLOS Genet 2011, 7:e1001264. 
12. Xiong $\mathrm{CH}$, Xia YL, Zheng P, Shi SH, Wang CS: Developmental stagespecific gene expression profiling for a medicinal fungus Cordyceps militaris. Mycology 2010, 1:25-66.

13. Galagan JE, Calvo SE, Borkovich KA, Selker EU, Read ND, Jaffe D, FitzHugh W, Ma LJ, Smirnov S, Purcell S, Rehman B, Elkins T, Engels R, Wang S, Nielsen CB, Butler J, Endrizzi M, Qui D, lanakiev P, Bell-Pedersen D, Nelson MA, Werner-Washburne M, Selitrennikoff CP, Kinsey JA, Braun EL, Zelter A, Schulte U, Kothe GO, Jedd G, Mewes W, et al: The genome sequence of the filamentous fungus Neurospora crassa. Nature 2003, 422:859-868

14. Ma LJ, van der Does HC, Borkovich KA, Coleman JJ, Daboussi MJ, Di Pietro A, Dufresne M, Freitag M, Grabherr M, Henrissat B, Houterman PM, Kang S, Shim WB, Woloshuk C, Xie X, Xu JR, Antoniw J, Baker SE, Bluhm BH, Breakspear A, Brown DW, Butchko RA, Chapman S, Coulson R, Coutinho PM, Danchin EG, Diener A, Gale LR, Gardiner DM, Goff S, et al: Comparative genomics reveals mobile pathogenicity chromosomes in Fusarium. Nature 2010, 464:367-373.

15. Kubicek CP, Herrera-Estrella A, Seidl-Seiboth V, Martinez DA, Druzhinina IS, Thon M, Zeilinger S, Casas-Flores S, Horwitz BA, Mukherjee PK, Mukherjee M, Kredics L, Alcaraz LD, Aerts A, Antal Z, Atanasova L, Cervantes-Badillo MG, Challacombe J, Chertkov O, McCluskey K, Coulpier F, Deshpande N, von Döhren H, Ebbole DJ, Esquivel-Naranjo EU, Fekete E, Flipphi M, Glaser F, Gómez-Rodríguez EY, Gruber S, et al: Comparative genome sequence analysis underscores mycoparasitism as the ancestral life style of Trichoderma. Genome Biol 2011, 12:R40.

16. Rajashekara G, Glasner JD, Glover DA, Splitter GA: Comparative wholegenome hybridization reveals genomic islands in Brucella species. J Bacteriol 2004, 186:5040-5051.

17. Fedorova ND, Khaldi N, Joardar VS, Maiti R, Amedeo P, Anderson MJ, Crabtree J, Silva JC, Badger JH, Albarraq A, Angiuoli S, Bussey H, Bowyer P, Cotty PJ, Dyer PS, Egan A, Galens K, Fraser-Liggett CM, Haas BJ, Inman JM, Kent R, Lemieux S, Malavazi I, Orvis J, Roemer T, Ronning CM, Sundaram JP, Sutton $G$, Turner G, Venter $\mathrm{JC}$, et al: Genomic islands in the pathogenic filamentous fungus Aspergillus fumigatus. PLoS Genet 2008, 4:e1000046.

18. McElwain JC, Punyasena SW: Mass extinction events and the plant fossil record. Trends Ecol Evol 2007, 22:548-557.

19. Cuomo CA, Güldener U, Xu JR, Trail F, Turgeon BG, Di Pietro A, Walton JD, Ma LJ, Baker SE, Rep M, Adam G, Antoniw J, Baldwin T, Calvo S, Chang YL, Decaprio D, Gale LR, Gnerre S, Goswami RS, Hammond-Kosack K, Harris LJ, Hilburn K, Kennell JC, Kroken S, Magnuson JK, Mannhaupt G, Mauceli E, Mewes HW, Mitterbauer R, Muehlbauer G, et al: The Fusarium graminearum genome reveals a link between localized polymorphism and pathogen specialization. Science 2007, 317:1400-1402.

20. Galagan JE, Selker EU: RIP: The evolutionary cost of genome defense. Trends Genet 2004, 20:417-423.

21. Screen SE, St Leger RJ: Cloning, expression, and substrate specificity of a fungal chymotrypsin. Evidence for lateral gene transfer from an actinomycete bacterium. J Biol Chem 2000, 275:6689-6694.

22. Coetzer TH, Goldring JP, Huson LE: Oligopeptidase B: a processing peptidase involved in pathogenesis. Biochimie 2008, 90:336-344.

23. Chong SL, Battaglia E, Coutinho PM, Henrissat B, Tenkanen M, de Vries RP: The a-glucuronidase Agu1 from Schizophyllum commune is a member of a novel glycoside hydrolase family (GH115). Appl Microbiol Biotechnol 2011, 90:1323-1332.

24. Duan ZB, Shang Y, Gao Q, Zheng P, Wang CS: A phosphoketolase Mpk1 of bacterial origin is adaptively required for full virulence in the insectpathogenic fungus Metarhizium anisopliae. Environ Microbiol 2009, 11:2351-2360.

25. Crešnar $B$, Petrič S: Cytochrome P450 enzymes in the fungal kingdom. Biochim Biophys Acta 2011, 1814:29-35.

26. Keller NP, Turner G, Bennett JW: Fungal secondary metabolism - from biochemistry to genomics. Nat Rev Microbiol 2005, 3:937-947.

27. Jarrold SL, Moore D, Potter U, Charnley AK: The contribution of surface waxes to pre-penetration growth of an entomopathogenic fungus on host cuticle. Mycol Res 2007, 111:240-249.

28. Morschhauser J: Regulation of multidrug resistance in pathogenic fungi. Fungal Genet Biol 2010, 47:94-106.

29. Luz C, Netto MC, Rocha LF: In vitro susceptibility to fungicides by invertebrate-pathogenic and saprobic fungi. Mycopathologia 2007, 164:39-47.
30. Kulkarni RD, Thon MR, Pan H, Dean RA: Novel G-protein-coupled receptorlike proteins in the plant pathogenic fungus Magnaporthe grisea. Genome Biol 2005, 6:R24.

31. Hejtmanek M, Hejtmankova N: Teleomorphs and mating types in Trichophyton mentagrophytes complex. Acta Univ Palacki Olomuc Fac Med 1989, 123:11-33.

32. Lennon MB, Suhadolnik RJ: Biosynthesis of 3'-deoxyadenosine by Cordyceps militaris. Mechanism of reduction. Biochim Biophys Acta 1976, 425:532-536.

33. Reichard P: Ribonucleotide reductases: substrate specificity by allostery. Biochem Biophys Res Commun 2010, 396:19-23.

34. Liu M, Panaccione DG, Schardl CL: Phylogenetic analyses reveal monophyletic origin of the ergot alkaloid gene dmaW in fungi. Evol Bioinform 2009, 5:15-30.

35. Brown DW, McCormick SP, Alexander NJ, Proctor RH, Desjardins AE: A genetic and biochemical approach to study trichothecene diversity in Fusarium sporotrichioides and Fusarium graminearum. Fungal Genet Biol 2001, 32:121-133.

36. Pöggeler S, Nowrousian M, Ringelberg C, Loros JJ, Dunlap JC, Kuck U: Microarray and real-time PCR analyses reveal mating type-dependent gene expression in a homothallic fungus. Mol Genet Genomics 2006, 275:492-503.

37. Kamata N, Sato H, Shimazu M: Seasonal changes in the infection of pupae of the beech caterpillar, Quadricalcarifera punctatella (Motsch.) (Lep., Notodontidae), by Cordyceps militaris Link (Clavicipitales, Clavicipitaceae) in the soil of the Japanese beech forest. J Appl Entomol 1997, 121:17-21

38. Clarkson JP, Staveley J, Phelps K, Young CS, Whipps JM: Ascospore release and survival in Sclerotinia sclerotiorum. Mycol Res 2003, 107:213-222.

39. Ohta Y, Lee JB, Hayashi K, Fujita A, Park DK, Hayashi T: In vivo antiinfluenza virus activity of an immunomodulatory acidic polysaccharide isolated from Cordyceps militaris grown on germinated soybeans. J Agric Food Chem 2007, 55:10194-10199.

40. Sung GH, Poinar GO Jr, Spatafora JW: The oldest fossil evidence of animal parasitism by fungi supports a Cretaceous diversification of fungalarthropod symbioses. Mol Phylogenet Evol 2008, 49:495-502.

41. Wang CS, St Leger RJ: The Metarhizium anisopliae perilipin homolog MPL1 regulates lipid metabolism, appressorial turgor pressure, and virulence. J Biol Chem 2007, 282:21110-21115.

42. Wang CS, Duan ZB, St Leger RJ: MOS1 osmosensor of Metarhizium anisopliae is required for adaptation to insect host hemolymph. Eukaryot Cell 2008, 7:302-309.

43. Wang CS, St Leger RJ: A collagenous protective coat enables Metarhizium anisopliae to evade insect immune responses. Proc Natl Acad Sci USA 2006, 103:6647-6652

44. Wang CS, St Leger RJ: The MAD1 adhesin of Metarhizium anisopliae links adhesion with blastospore production and virulence to insects, and the MAD2 adhesin enables attachment to plants. Eukaryot Cell 2007, 6:808-816

45. Freitag M, Williams RL, Kothe GO, Selker EU: A cytosine methyltransferase homologue is essential for repeat-induced point mutation in Neurospora crassa. Proc Natl Acad Sci USA 2002, 99:8802-8807.

46. Montiel MD, Lee HA, Archer DB: Evidence of RIP (repeat-induced point mutation) in transposase sequences of Aspergillus oryzae. Fungal Genet Biol 2006, 43:439-445.

47. Long $M$, Betran $E$, Thornton $K$, Wang $W$ : The origin of new genes: glimpses from the young and old. Nat Rev Genet 2003, 4:865-875.

48. Ekman D, Elofsson A: Identifying and quantifying orphan protein sequences in fungi. J Mol Biol 2010, 396:396-405.

49. Ohm RA, de Jong JF, Lugones $L G$, Aerts A, Kothe E, Stajich JE, de Vries RP, Record E, Levasseur A, Baker SE, Bartholomew KA, Coutinho PM, Erdmann S, Fowler TJ, Gathman AC, Lombard V, Henrissat B, Knabe N, Kües U, Lilly WW, Lindquist E, Lucas S, Magnuson JK, Piumi F, Raudaskoski M, Salamov A, Schmutz J, Schwarze FW, vanKuyk PA, Horton JS, Grigoriev IV, et al: Genome sequence of the model mushroom Schizophyllum commune. Nat Biotechnol 2010, 28:957-963.

50. Corson TW, Crews CM: Molecular understanding and modern application of traditional medicines: triumphs and trials. Cell 2007, 130:769-774.

51. Yan WJ, Li TH, Lin QY, Song B, Jiang ZD: Safety assessment of Cordyceps guangdongensis. Food Chem Toxicol 2010, 48:3080-3084. 
52. Heitman J: Evolution of eukaryotic microbial pathogens via covert sexual reproduction. Cell Host Microbe 2010, 8:86-99.

53. Keeney S: Mechanism and control of meiotic recombination initiation. Curr Top Dev Biol 2001, 52:1-53

54. Masson JY, West SC: The Rad51 and Dmc1 recombinases: a non-identical twin relationship. Trends Biochem Sci 2001, 26:131-136.

55. Fisher RP: Secrets of a double agent: CDK7 in cell-cycle control and transcription. J Cell Sci 2005, 118:5171-5180.

56. Schatz MC, Delcher AL, Salzberg SL: Assembly of large genomes using second-generation sequencing. Genome Res 2010, 20:1165-1173.

57. Ter-Hovhannisyan V, Lomsadze A, Chernoff YO, Borodovsky M: Gene prediction in novel fungal genomes using an $a b$ initio algorithm with unsupervised training. Genome Res 2008, 18:1979-1990.

58. Zhang Z, Carriero N, Zheng D, Karro J, Harrison PM, Gerstein M: PseudoPipe: an automated pseudogene identification pipeline. Bioinformatics 2006, 22:1437-1439.

59. SignalP 3.0 Server.. [http://www.cbs.dtu.dk/services/SignalP/].

60. RepeastMasker Server.. [http://www.repeatmasker.org/].

61. Benson G: Tandem repeats finder: a program to analyze DNA sequences. Nucleic Acids Res 1999, 27:573-580.

62. Kohany O, Gentles AJ, Hankus L, Jurka J: Annotation, submission and screening of repetitive elements in Repbase: RepbaseSubmitter and Censor. BMC Bioinformatics 2006, 7:474.

63. Schmidt HA, Strimmer K, Vingron M, von Haeseler A: TREE-PUZZLE: maximum likelihood phylogenetic analysis using quartets and parallel computing. Bioinformatics 2002, 18:502-504.

64. Taylor JW, Berbee M: Dating divergences in the Fungal Tree of Life: review and new analyses. Mycologia 2006, 98:838-849.

65. Lucking R, Huhndorf S, Pfister DH, Plata ER, Lumbsch HT: Fungi evolved right on track. Mycologia 2009, 101:810-822.

66. InterPro Protein Sequence Analysis \& Classification.. [http://www.ebi.ac. uk/interpro/].

67. Pfam Database.. [http://pfam.sanger.ac.uk]].

68. Schomburg D, Schomburg IL: Enzyme databases. Methods Mol Biol 2010, 609:113-128.

69. Cytochrome P450 Server.. [http://drnelson.uthsc.edu/CytochromeP450. html].

70. Saier MH Jr, Tran CV, Barabote RD: TCDB: the Transporter Classification Database for membrane transport protein analyses and information. Nucleic Acids Res 2006, 34:D181-186.

71. GPCR Database.. [http://www.gpcr.org/7tm/].

72. PHI-base: Pathogen-Host Interactions.. [http://www.phi-base.org]

73. Kanehisa M, Goto S, Kawashima S, Nakaya A: The KEGG databases at GenomeNet. Nucleic Acids Res 2002, 30:42-46.

74. SMURF.. [http://www.jcvi.org/smurf/index.php].

75. Anand S, Prasad MV, Yadav G, Kumar N, Shehara J, Ansari MZ, Mohanty D: SBSPKS: structure based sequence analysis of polyketide synthases. Nucleic Acids Res 2010, 38:W487-496.

76. Kim YT, Lee YR, Jin J, Han KH, Kim H, Kim JC, Lee T, Yun SH, Lee YW: Two different polyketide synthase genes are required for synthesis of zearalenone in Gibberella zeae. Mol Microbiol 2005, 58:1102-1113.

77. Dao HP, Mathieu F, Lebrihi A: Two primer pairs to detect OTA producers by PCR method. Int J Food Microbiol 2005, 104:61-67.

78. Artigot MP, Loiseau N, Laffitte J, Mas-Reguieg L, Tadrist S, Oswald IP, Puel O: Molecular cloning and functional characterization of two CYP619 cytochrome P450s involved in biosynthesis of patulin in Aspergillus clavatus. Microbiology 2009, 155:1738-1747.

79. Proctor RH, Desjardins AE, Plattner RD, Hohn TM: A polyketide synthase gene required for biosynthesis of fumonisin mycotoxins in Gibberella fujikuroi mating population A. Fungal Genet Biol 1999, 27:100-112.

80. Shimizu T, Kinoshita H, Ishihara S, Sakai K, Nagai S, Nihira T: Polyketide synthase gene responsible for citrinin biosynthesis in Monascus purpureus. Appl Environ Microbiol 2005, 71:3453-3457.

81. Ehrlich KC, Chang PK, Yu J, Cotty PJ: Aflatoxin biosynthesis cluster gene cypA is required for $\mathrm{G}$ aflatoxin formation. Appl Environ Microbiol 2004, 70:6518-6524.

82. $\mathrm{Yu} \mathrm{JH}$, Leonard TJ: Sterigmatocystin biosynthesis in Aspergillus nidulans requires a novel type I polyketide synthase. J Bacteriol 1995, 177:4792-4800
83. Gardiner DM, Howlett BJ: Bioinformatic and expression analysis of the putative gliotoxin biosynthetic gene cluster of Aspergillus fumigatus. FEMS Microbiol Lett 2005, 248:241-248.

84. Haese A, Schubert M, Herrmann M, Zocher R: Molecular characterization of the enniatin synthetase gene encoding a multifunctional enzyme catalyzing $\mathrm{N}$-methyldepsipeptide formation in Fusarium scirpi. Mol Microbiol 1993, 7:905-914

85. Scott-Craig JS, Panaccione DG, Pocard JA, Walton JD: The cyclic peptide synthetase catalyzing HC-toxin production in the filamentous fungus Cochliobolus carbonum is encoded by a 15.7-kilobase open reading frame. J Biol Chem 1992, 267:26044-26049.

86. Weber G, Schörgendorfer K, Schneider-Scherzer E, Leitner E: The peptide synthetase catalyzing cyclosporine production in Tolypocladium niveum is encoded by a giant 45.8-kilobase open reading frame. Curr Genet 1994 26:120-125.

87. Brenner S, Johnson M, Bridgham J, Golda G, Lloyd DH, Johnson D, Luo S, McCurdy S, Foy M, Ewan M, Roth R, George D, Eletr S, Albrecht G, Vermaas E, Williams SR, Moon K, Burcham T, Pallas M, DuBridge RB, Kirchner J, Fearon K, Mao J, Corcoran K: Gene expression analysis by massively parallel signature sequencing (MPSS) on microbead arrays. Nat Biotechnol 2000, 18:630-634

88. Bruno VM, Wang Z, Marjani SL, Euskirchen GM, Martin J, Sherlock G, Snyder M: Comprehensive annotation of the transcriptome of the human fungal pathogen Candida albicans using RNA-seq. Genome Res 2010, 20:1451-1458.

89. Benjamini Y, Yekutieli D: The control of the false discovery rate in multiple testing under dependency. Ann Stat 2001, 29:1165-1188.

doi:10.1186/gb-2011-12-11-r116

Cite this article as: Zheng et al:: Genome sequence of the insect pathogenic fungus Cordyceps militaris, a valued traditional chinese medicine. Genome Biology 2011 12:R116.

\section{Submit your next manuscript to BioMed Central and take full advantage of:}

- Convenient online submission

- Thorough peer review

- No space constraints or color figure charges

- Immediate publication on acceptance

- Inclusion in PubMed, CAS, Scopus and Google Scholar

- Research which is freely available for redistribution

Submit your manuscript at www.biomedcentral.com/submit
Ciomed Central 